\title{
DISTRIBUTION AND HABITAT PREFERENCES OF THE INVASIVE ALIEN ROSA RUGOSA (ROSACEAE) IN ARCHIPELAGO SEA NATIONAL PARK, SW FINLAND
}

\author{
Panu Kunttu ${ }^{1} \&$ Sanna-Mari Kunttu
}

\begin{abstract}
Rosa rugosa Thunb. is an invasive alien plant which was introduced from East Asia to Europe in the $19^{\text {th }}$ century. Over the decades it has spread along the Finnish coast, including the archipelagos of the Baltic Sea. The shrub grows particularly along sandy beaches, stony shores and seashore meadows. There it leads to serious changes in coastal ecosystems and replaces native plant species. This paper presents the distribution and habitat preferences of $R$. rugosa in and near Archipelago Sea National Park in SW Finland. We identified 205 stands in the study area, 58\% of them in protected areas. Systematic inventories do not cover the whole study area, so it is likely that dozens of stands remain undiscovered. Stands of the species were concentrated on the outer islands, where the archipelago's most characteristic flora and fauna occur. Data on the shore type and area of all stands were collected. The total area covered by $R$. rugosa was $7277 \mathrm{~m}^{2}$ before control work started in 2008 . The largest stand occurred on Örö Island, covering $c a 2500 \mathrm{~m}^{2}$ before its eradication, and the median size of all stands was $6 \mathrm{~m}^{2}$. Five of the six largest stands were on sandy beach, but stony shore was the most common habitat type.
\end{abstract}

Key words: Baltic Sea, coastal habitats, invaded area, non-native species, population size

Panu Kunttu, University of Eastern Finland, School of Forest Sciences, P.O. Box 111, FI-80101, Joensuu, Finland; e-mail: panu.kunttu@iki.fi

Sanna-Mari Kunttu, Hertsbölevägen 8 H, FI-25900 Dalsbruk, Finland; e-mail: sanna-mari.kunttu@iki.fi

\section{INTRODUCTION}

Invasive alien species have spread from their natural ranges to new areas as a result of human activities and are considered to have negative ecological impacts (terminology is discussed in Colautti \& MacIsaac 2004). Invasive species have crossed geographical barriers that historically were natural borders of species ranges (Scalera et al. 2012). Invasive species have negative ecological, economic or social effects in the introduced range. The most harmful invasive species can create widespread and irreversible problems for entire ecosystems or regions.

Invasive alien plants have become a significant problem for biological conservation and ecosystem management, due to their negative effects on biodiversity (Nentwig 2007; Hejda et al. 2009; Goodenough 2010; Scalera et al. 2012). Along with habitat loss and degradation, invasive species

\footnotetext{
1 Corresponding author
}

are one of the most serious threats to biodiversity worldwide (Walker \& Steffen 1997; McGeoch et al. 2010). Introduced species are drivers of several extinctions, especially on islands (Veitch et al. 2011).

In Finland, Rosa rugosa Thunb. has been classified as a very harmful invasive plant due to its negative effects on native species and natural habitats (Niemivuo-Lahti 2012). The purpose of this study was to identify the extent to which $R$. rugosa occurs in Archipelago Sea National Park, SW Finland. We focused on its distribution, habitat preferences, stand size and control. This is the first regional review of this species in Finland.

\section{DISTRIBUTION OF ROSA RUGOSA}

Rosa rugosa is a tall shrub originating from East Asia, where it occurs along sandy and gravelly coasts of the Pacific Ocean in Japan, Korea, China 
and Russia. The species was introduced to gardens in Europe as early as 1796 (Bruun 2005). It has become widely naturalized in Europe and North America from ornamental plantings, most often as a garden escapee (Hill et al. 2010). It is naturalized especially in parts of Northern and Northwestern Europe and is regarded as invasive along the coasts of the northwestern European Atlantic, North Sea and Baltic Sea, including the Baltic countries, Fennoscandia, northern Germany, Great Britain and Poland (Bruun 2005; Kelager et al. 2013). Rosa rugosa has been classified as an established or at least already naturalized invasive species in 22 European countries (Bruun 2005; Essl 2006; Weidema 2006).

The first Finnish record of naturalized $R$. rugosa is from 1919, after which the plant began to spread successfully in the 1930s (Erkamo 1949). Rosa rugosa occurs throughout the coastal area and the archipelago of Finland to Bothnian Bay, including the Åland Islands (Hæggström \& Hæggström 2010; Lampinen \& Lahti 2016). On the southern coast, on the archipelago of the Gulf of Finland and the Archipelago Sea it is most common. The situation is worst on the Hanko Peninsula on the southern coast of Finland, where the species has colonized several hectares of sandy beaches (Skytén 1978; Aspelund \& Ryttäri 2010).

\section{RoSA RUGOSA AS A THREAT TO NATIVE SPECIES} AND HABITATS

In Europe, $R$. rugosa mainly invades coastal habitats, especially sand dunes, but also vegetated shingle, grassland and low scrub, verges and waste ground. It can also become invasive in inland habitats such as Calluna heath (Isermann 2008b; Kollmann et al. 2009; Thiele et al. 2009). Rosa rugosa is a great threat to coastal ecosystems because it replaces native plant species and changes the composition of habitat types and suppresses natural vegetation of high conservation value (Kollmann et al. 2009; Thiele et al. 2010, 2011).

In Northwestern Europe, especially on shores of the North Sea and Baltic Sea, R. rugosa has been a major invader of sand dunes and is a significant problem in dune conservation (Reddersen 2006;
Weidema et al. 2007; Isermann 2008a, b; Jørgensen \& Kollmann 2009). Rosa rugosa is among the 100 worst alien species in Europe (Essl 2006).

In contrast to North Sea dunes and more southern Baltic dune areas, in Finland R. rugosa is established on the shore, where its invasiveness was noted early (Jalas 1965). In these habitats it threatens native vegetation and leads to reduced diversity of native species (e.g., Skytén 1978; Ryttäri et al. 2006; Ryttäri et al. 2014). Shores support specialized flora and fauna and several red-listed species. Rosa rugosa is considered a threat to 21 species living on sandy beaches in Finland, mainly insects and vascular plants (Rassi et al. 2010; Niemivuo-Lahti 2012). Sandy beaches, dunes, shrub heaths and seashore meadows are particularly susceptible to overgrowth, and R. rugosa is one factor of this phenomenon. These habitats are classified as threatened in Finland (Raunio et al. 2008) and are listed in the EU's Natura 2000 program. Many of these habitats cover only small areas and are fairly rare.

\section{STUDY AREA}

The study area, Archipelago Sea National Park and its surroundings, is on the Baltic Sea in SW Finland (approx. $60^{\circ} \mathrm{N}, 22^{\circ} \mathrm{E}$; Fig. 1). The area is in the hemiboreal zone (Ahti et al. 1968) and belongs to Kimitoön and Pargas municipalities. This national park is also known as Archipelago National Park or South Western Archipelago National Park. The park and its unprotected surroundings cover $151 \mathrm{~km}^{2}$ of land and $2896 \mathrm{~km}^{2}$ of sea, and contain 8384 islands or skerries (size at least $100 \mathrm{~m}^{2}$ ). Only $23 \%$ of the land area is protected. The rest, inside the boundary shown in Figure 1, is unprotected privately owned land.

Archipelago Sea National Park is unique due to its glaciation-affected geology, mosaic landscape and historical land uses. Nowhere else is there such a similar density of islands, and the number of species and habitat types is the highest in Finland. For example, 45 habitat types of the Natura 2000 program have been found in this area (Lindgren 2000). The number of red-listed species is exceptionally high, with 467 species 


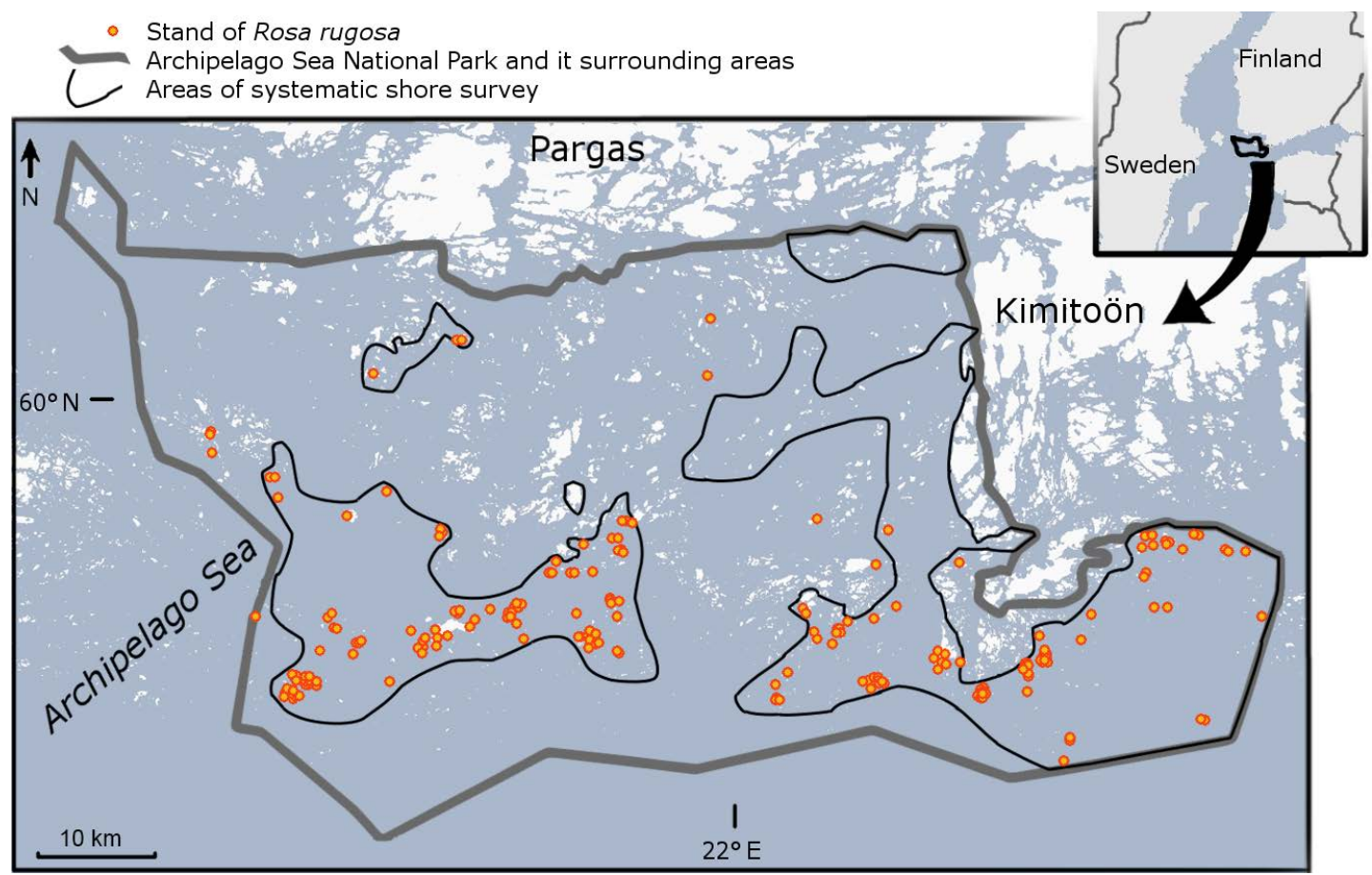

Fig. 1. Study area in SW Finland, areas of systematic shore survey, and distribution of stands of the invasive alien Rosa rugosa Thunb.

(Kunttu et al. 2014). Many habitat types cover small areas, so invasive species can have ruinous impacts on their biodiversity.

In the outer archipelago, the growing season lasts 191 days on average and the effective temperature sum (the sum of the positive differences between diurnal mean temperature and 5 degrees Celsius) is $1250-1300$ (Kersalo \& Pirinen 2009). The mean annual temperature is $+6.5^{\circ} \mathrm{C}$, and annual precipitation $549 \mathrm{~mm}$. The prevailing winds blow from the southwest $(20 \%)$, and the average wind speed is $7.1 \mathrm{~m} / \mathrm{s}$ (Pirinen et al. 2012). The islands consist mainly of gneiss and granite bedrock with soil layers of moraine, sand or gravel deposition (http://en.gtk.fi). The highest point of the study area is $42 \mathrm{~m}$ a.s.l.

\section{MATERIAL AND METHODS}

The study is based mainly on systematic inventories of certain archipelago areas and partly on occasional records made during other activities. A systematic inventory of the whole study area would have been a huge task due to the very high number of islands and skerries. We carried out systematic inventories of shores on 1150 islands and skerries during 2014-2016 (Fig. 1).

Other data on the occurrence of $R$. rugosa were collected from notes of Metsähallitus Parks \& Wildlife Finland (manager of the National Park), Kastikka and Hatikka databases made by the Finnish Museum of Natural History, inventory reports and information from individual naturalists (Ikonen et al. 2009; von Numers 2011; Lampinen \& Lahti 2016; http://fieldjournal. org; http://karttapalvelu.lounaispaikka.fi). Ornithologists have mapped the occurrence of $R$. rugosa on Jurmo Island. We used all data available from these sources up to July 2016 or collected in the field.

All records contain the location of the station, given with coordinates of the World Geodetic System (WGS84). Accurate information on stand size, habitat type and possible control measures are given. Yards and gardens were excluded from this study. The habitat types used in this study were sandy beach, stony shore (including also gravel, shingle and boulder shores), seashore meadows and dry meadows, rock outcrops, and dwarf shrub heaths (Raunio et al. 2008). We used the 
non-parametric Kruskal-Wallis test (SPSS Statistics 21) to test whether the mean size of stands differs significantly between shore types.

\section{RESULTS}

Stands of $R$. rugosa were found at 205 sites in Archipelago Sea National Park and its surroundings (Fig. 1 \& Appendix). Stand density was highest through the outermost part of the study area (Fig. 1). The most frequent stand loci were situated in the southwestern, southern and southeastern parts of the study area, which are part of an extensive moraine ridge.

Ninety-five stands were recorded in the national park, 23 were recorded in a privately owned nature reserve, and 87 records were from unprotected private land.

The two largest stands were on Örö ( $c a 2500 \mathrm{~m}^{2}$ and $2000 \mathrm{~m}^{2}$ ), though control measures eradicated one of them and significantly reduced the other since the time of those records. The largest stand was already known in 1963, when it covered only a few square meters (Kastikka database). The next largest stands were noted at Örskärs ören near Utö (304 m², Fig. 3), Stengrundet (126 m²), Långlandet $\left(100 \mathrm{~m}^{2}\right)$ and Estrevlarna $\left(100 \mathrm{~m}^{2}\right)$ (APPENDIX). The six largest stands account for $71 \%$ of the total area covered by $R$. rugosa in the study area. The distribution of the number of stands, and their total area (given in 6 size classes), are presented in Figure 2. Most of the stands were about a meter high, and the tallest stand was two meters high (Fig. 3).

Stand size and habitat type were known for 201 stands. Their total area was $7277 \mathrm{~m}^{2}$ (median $6 \mathrm{~m}^{2}$, range $0.5-2500 \mathrm{~m}^{2}$ ). In careful inventories in 2014 and 2016 only four old stands were not found.

The sizes given for the two largest stands on Örö Island are only estimates, not precise measurements. The estimates of their sizes before control measures were made afterwards; these are rather small stands now. All other stands for which size information is given were rigorously

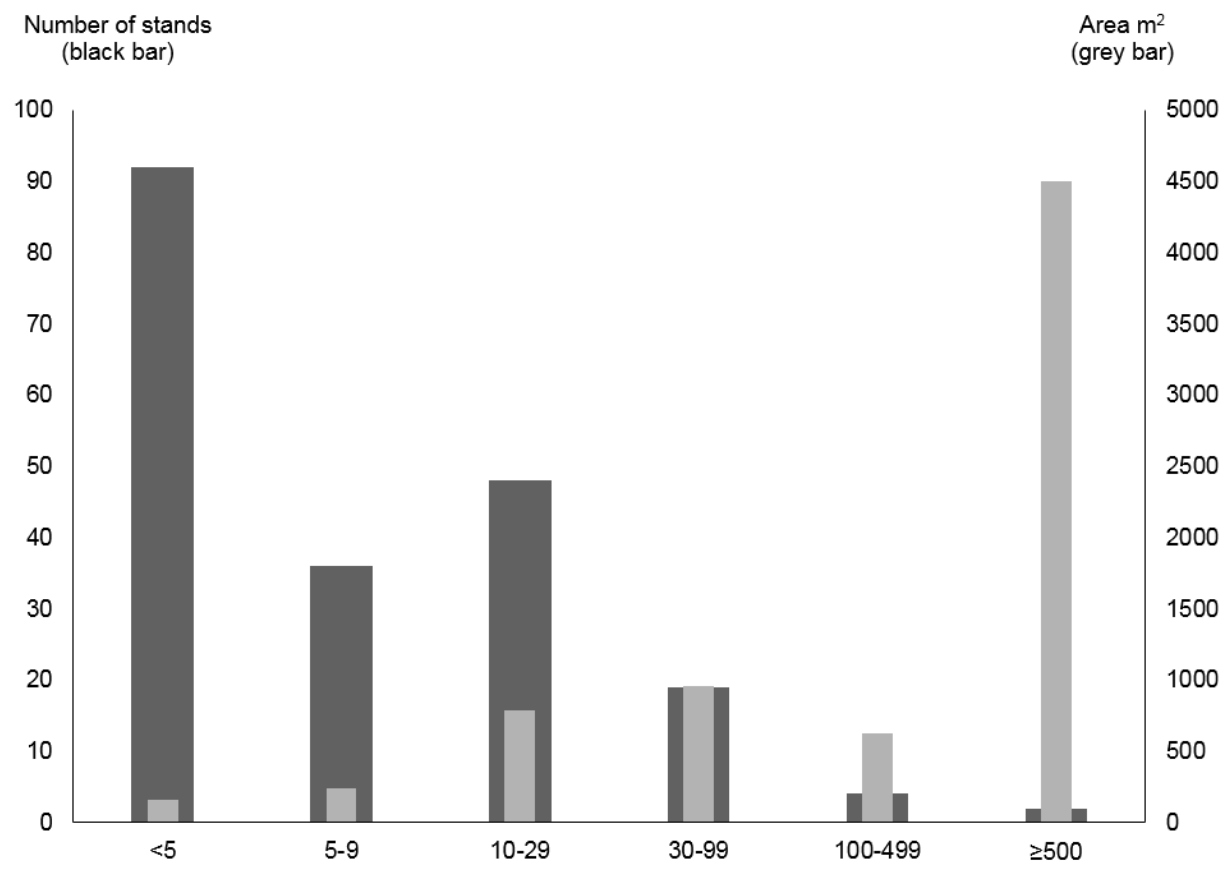

Size class $\left(\mathrm{m}^{2}\right)$

Fig. 2. Distribution of number of stands $(\mathrm{N}=201)$ of Rosa rugosa Thunb., and their total area given in six size classes. 

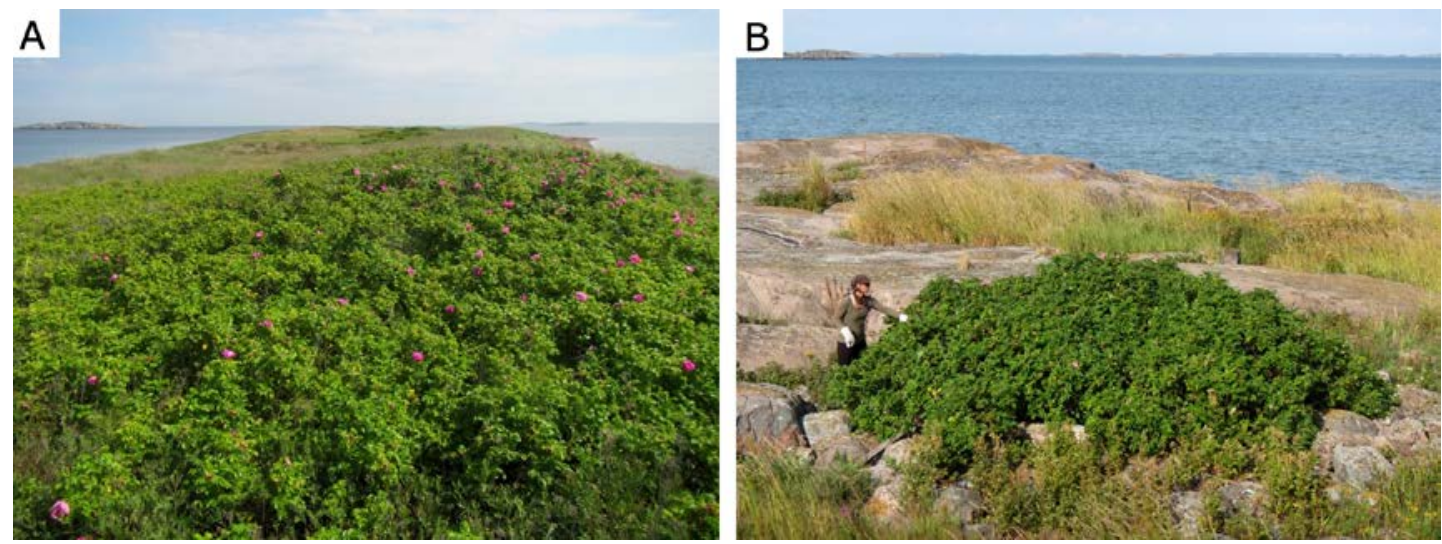

Fig. 3. One of the largest $\left(\mathrm{A}-304 \mathrm{~m}^{2}\right)$ and one of the tallest $(\mathrm{B}-2 \mathrm{~m})$ stands of the invasive alien Rosa rugosa Thunb. in the study area. Photo P. Kunttu.

measured. The number of stands and their sizes are shown, by habitat type, in Table 1 .

Rosa rugosa occurs in many different habitats in Archipelago Sea National Park (Fig. 4). The most common habitat type was stony shore, where $47 \%$ of the stands were found, but most of the colonized area as well as the largest stands were on sandy beaches (Table 1). The mean size of stands differed significantly between shore types (Kruskal-Wallis test, Chi-square $=26.868, p>0.001)$. The typical habitats of $R$. rugosa in Archipelago Sea National Park are presented in Figure 4.

Control measures and removal were performed at 45 sites (22\% of all sites), but only eight stands can be considered eradicated (APPENDIX). Volunteers did most of this control work (38 cases). Based on their size and habitat type, the stand eradications on Örö have been the most important ones. Several control measures have been used: manual uprooting, mechanical uprooting, herbicide treatment, and covering with a tarpaulin. The most typical control method has been manual uprooting and collection of all stems, rhizomes and roots to leave on rocks to dry; in some cases the plant material was burned. This type of control needs to be repeated for many years, and some roots may remain in the ground. Covering stands with tarpaulins for several years or shrivelling shrubs by ripping the leaves off may frequently prove to be more effective as control measures.

\section{Discussion}

The area covered by $R$. rugosa in Archipelago Sea National Park is not as large as at some other localities in the Baltic region (e.g., Kollmann et al. 2009; Aspelund \& Ryttäri 2010), but there are many stands and they occur throughout the study

Table 1. Number of stands of the invasive alien Rosa rugosa Thunb. and their area according to habitat type; - no information available, stands not found.

\begin{tabular}{lcccc}
\hline Habitat type & Number of stands & Total area $\left(\mathrm{m}^{2}\right)$ & Median size $\left(\mathrm{m}^{2}\right)$ & Range $\left(\mathrm{m}^{2}\right)$ \\
\hline Sandy beach & 19 & 5356 & 16 & $0.5-2500$ \\
Stony shore & 94 & 737 & 4.5 & $0.5-58$ \\
Seashore meadow & 50 & 982 & 13 & $0.5-100$ \\
and dry meadow & 9 & 60 & 6 & $0.5-17$ \\
Dwarf shrub heath & 29 & 142 & 1 & $0.5-30$ \\
Rock outcrops & 4 & - & - & - \\
Unknown & 205 & 7277 & 6 & $0.5-2500$ \\
\hline Total & & & & \\
\hline
\end{tabular}



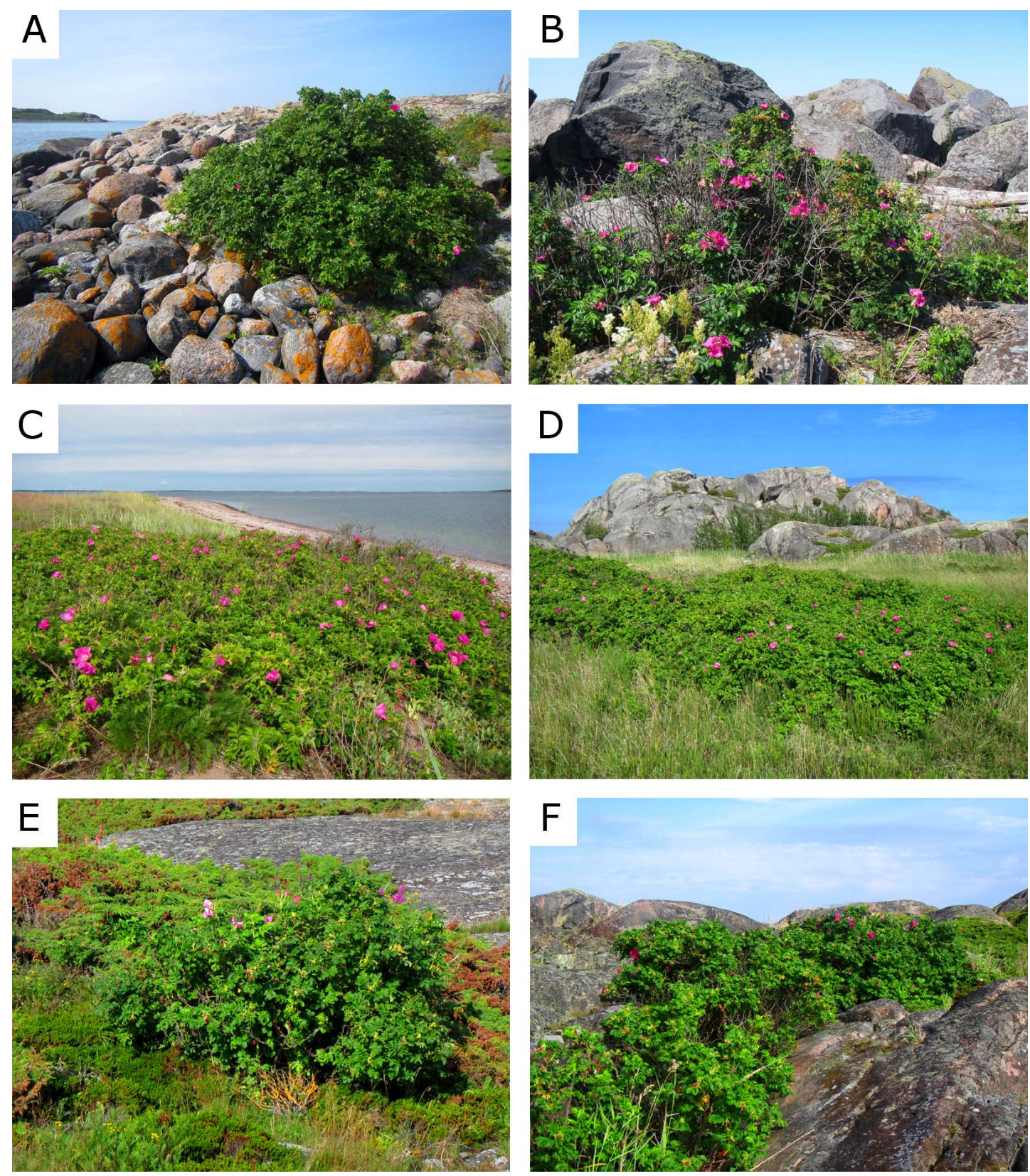

Fig. 4. Typical habitats of the invasive alien Rosa rugosa Thunb. in Archipelago Sea National Park and its surroundings: A - stony shore (middle-size stones), B - stony shore (boulders), C - sandy beach, D - seashore meadow, E - dwarf shrub heath, F - rock outcrop on seashore. Photo P. Kunttu.

area, so their potential for dispersal is high. Because there are many stands, and many small ones, now is the time to remove $R$. rugosa before the stands grow much larger and become really problematic to remove. The islands and skerries are near each other, facilitating seed dispersal. In addition to the naturalized stands, $R$. rugosa is still used in gardens on many inhabited islands. 
There are no data on the origin of the $R$. rugosa seeds coming to the archipelago. Presumably there are many origins: inhabited islands of this area, mainland Finland, the Hanko Peninsula, and the coasts of Estonia and Sweden. Rosa rugosa hips are extraordinarily buoyant and can float for up to 40 weeks in both freshwater and seawater. The seeds are also buoyant for several weeks on their own (Jessen 1958). They are dispersed by birds or waves, so their origin may be very far off on mainland Finland, inland, or overseas from Estonia, Russia or Sweden where R. rugosa is common (Tzvelev 2007; Ööpik et al. 2008; Tyler et al. 2015). The general circulation in the Baltic Sea is counterclockwise: northward along its eastern boundary and southward along the western one; there is no strong permanent sea current. Southwestern winds are predominant on Finland's southwest coast. The flow velocity of surface water in the Baltic Sea is $5-10 \mathrm{~cm} / \mathrm{s}$, that is, several kilometers per day (Leppäranta \& Myrberg 2009).

The records of $R$. rugosa are concentrated in the outermost part of the study area. Probably one reason for this is that there are more suitable shore habitats there: the islands are flat, sandy or stony, and almost barren of forests. Basically, $R$. rugosa is able to grow anywhere perennial vegetation occurs.

The flora of the Archipelago Sea was studied widely by several researchers without documentation of the occurrence of $R$. rugosa (e.g., Olsoni 1946; Eklund 1958; Skult 1960; Stjernberg et al. 1974). The first records of naturalized $R$. rugosa in the study area were made in 1963 on Örö Island and in 1967 on Kuggskär Island. Much later, von Numers (2011) found R. rugosa on 17 islands which Eklund (1958) had studied.

Several areas have been systematically inventoried (Fig. 1), so the occurrences of R. rugosa in these areas are well known. For example, the central and northeastern parts of the study area are almost free of $R$. rugosa. Many areas in the outer part yielded numerous stands (Fig. 1). A large part of the most important area of its potential occurrence is now surveyed. There are still some unresearched island groups with gravel and shingle shores, part of the Salpausselkä moraine ridge formation, which could contain stands. Probably there are at least dozens of undiscovered stands in the national park and its surroundings, but they are not likely to be larger than those found so far. Unfortunately, new stands will form in the future, making control work very challenging.

Altogether $46 \%$ of the stands were found in the national park, although only a minority of the land in the study belongs to the national park. The reason for this finding is that the park's islands are in the outer part, which apparently is the most favorable area for $R$. rugosa due to the character of its habitats.

Five of the six largest stands grew on sandy beach. This shows that $R$. rugosa can spread vegetatively more easily on sandy beach than for example on rock outcrops on seashores. This makes it possible to prioritize control measures. For example, on the sandy beaches of Jurmo Island, $R$. rugosa threatens the red-listed species Ammophila arenaria (L.) Link. (EN) and Salsola kali L. subsp. kali (EN), and the sandy beaches of Örö also have Armeria maritima subsp. elongata (Hoffm.) Bonnier (EN), Dianthus arenarius subsp. borussicus Vierh. (EN) and Elymus farctus subsp. boreoali-atlanticus (Simonet \& Guin.) Melderis (VU) (Syrjänen 1995; Aspelund \& Ryttäri 2010; Kämäräinen 2013).

If the spread of $R$. rugosa is not successfully stopped, it will pose a serious threat to many native species and habitat types of the Archipelago Sea area. We already have examples of how it can become a severe problem for biodiversity in Finland (Ryttäri et al. 2006; Aspelund \& Ryttäri 2010). At risk are not only shore habitats but potentially also semi-natural habitats like shrub heaths and different kinds of meadows if eradication is not done in time. Prevention of dispersal through practical legislation and raised public awareness are needed to reduce the current ecological problems related to $R$. rugosa invasion.

ACKNOwledgements. Dr. Mikael von Numers ( $\AA$ bo Akademi), Dr. Mikael Nordström, Jouko Högmander, Esko Tainio and Matti Peltonen (Metsähallitus, Parks \& Wildlife Finland), Leena Lehtomaa (ELY-Centre in Varsinais-Suomi), Juha Kylänpää and Petri Vainio 
(The Ornithological Society of Turku) supplied valuable information on the occurrence of Rosa rugosa in Archipelago Sea National Park. Kaj Genberg (Kimitoön) helped in the fieldwork on the Rosala Archipelago. Otava Piha checked the English of an earlier version of the paper. Professor Johannes Kollmann (Technische Universität München) and one anonymous referee made valuable emendations to the manuscript. The Raija and Ossi Tuuliaisen Foundation financed the fieldwork period in 2016. All are warmly thanked.

\section{REFERENCES}

Ahtı T., Häмet-Ahtı L. \& Jalas J. 1968. Vegetation zones and their sections in northwestern Europe. Ann. Bot. Fenn. 5: 169-211.

AsPelund P. \& RytTÄRI T. 2010. The Japanese Rose threatens the flora and fauna of sandy seashores. Lutukka 26(1): 3-9 (in Finnish with English summary).

BruUn H. H. 2005. Biological flora of the British Isles. Rosa rugosa Thunb. Ex. Murray. J. Ecol. 93: 441-470.

Colautti R. I. \& MacIsaAC H. J. 2004. A neutral terminology to define 'invasive' species. Diversity and Distributions 10: $135-141$.

EKLUND O. 1958. Die Gefässpflanzenflora beiderseits Skiftet im Schärenarchipel Südwestfinnlands: Kirchspiele Korpo, Houtskär, Nagu, Iniö, Brändö, Kumlinge, Sottunga und Kökar. Bidrag Kännedom Finlands Natur Folk 101: 1-321.

ERKamo V. 1949. Rosa rugosa Thunb. ein für Europa neuer Neophyt. Archivum Soc. Vanamo 3: 123.

Essl F. 2006. Rosa rugosa. Species factsheet. Delivering Alien Invasive Species Inventories for Europe (DAISIE). [16 Dec. 2016] http://www.europe-aliens.org/speciesFactsheet.do?speciesId=14154.

Goodenough A. E. 2010. Are the ecological impacts of alien species misrepresented? A review of the 'native good, alien bad' philosophy. Community Ecol. 11: 13-21.

HÆgGström C.-A. \& HÆGgström E. 2010. Ålands flora. Privately published, Ekenäs.

HeJdA M., PyšEK P. \& JARošík V. 2009. Impact of invasive plants on the species richness, diversity and composition of invaded communities. J. Ecol. 97: 393-403.

Hill N., Beveridge L., Flynn A. \& Garbary D. J. 2010. Rosa rugosa as an invader of coastal sand dunes of Cape Breton Island and mainland of Nova Scotia. Can. Field Nat. 124(2): 151-158.

IKONEN I., KeKKI M. \& RäIKKÖNEN N. 2009. Jättiputki ja kurtturuusu kuriin Lounais-Suomessa. Lounais-Suomen Ympäristökeskuksen Raportteja 15: 1-51.

ISERMANN M. 2008a. Expansion of Rosa rugosa and Hippophaë rhamnoides in coastal grey dunes: effects at different spatial scales. Flora 203: 273-280.

ISERMANN M. 2008b. Classification and habitat characteristics of plant communities invaded by the non-native Rosa rugosa Thunb. in NW Europe. Phytocoenologia 38: 133-150.

Jalas J. 1965. Rosa rugosa Thunb. - Kurttulehtiruusu. In: JALAS J. (ed.), Suuri Kasvikirja. 2: 773-774. Otava, Helsinki.

JESSEN K. 1958. Om vandspredning af Rosa rugosa og andre arter af slægten. Bot. Tidsskr. 54: 353-366.

JøRGENSEN R. H. \& Kollmann J. 2009. Invasion of coastal dunes by the alien shrub Rosa rugosa is associated with roads, tracks and houses. Flora 204: 289-297.

KäMÄRÄINEN, H. 2013. The special flora of Örö Island, NW Gulf of Finland. Lutukka 29(3): 67-87 (in Finnish with English summary).

Kelager A., Pedersen J. S. \& Bruun H. H. 2013. Multiple introductions and no loss of genetic diversity: invasion history of Japanese Rose, Rosa rugosa, in Europe. Biol. Invasions 15:1125-1141.

Kersalo J. \& Pirinen P. (eds) 2009. The climate of Finnish regions. Finnish Meteorological Institute, Reports 8(2009): 1-185 (in Finnish with English summary).

Kollmann J., Jørgensen R. H., Roelsgaard J. \& SkovPetersen H. 2009. Establishment and clonal spread of the alien shrub Rosa rugosa in coastal dunes - A method for reconstructing and predicting invasion patterns. Landscape and Urban Planning 93: 194-200.

Kunttu P., Ryan V., Tolvanen P., Vilhunen S. \& Ormio H. 2014. Saaristomme on suojeltava. Esitys Saaristomeren, Tammisaaren saariston ja Itäisen Suomenlahden kansallispuistojen suojelun kehittämiseksi ja Porkkalan kansallispuiston perustamiseksi. WWF Suomen Raportteja 32: $1-63$.

LAMPINEN R. \& LAHTi T. 2016. Kasviatlas 2015. Helsingin yliopisto, Luonnontieteellinen keskusmuseo, Helsinki. [1 Aug. 2016]. http://www.luomus.fi/kasviatlas.

Leppäranta M. \& Myrberg K. 2009. Physical oceanography of the Baltic Sea. Springer-Praxis, Berlin Heidelberg.

LiNDGREN L. 2000. Island pastures. Metsähallitus and Edita, Helsinki.

McGeoch M. A., Butchart S. H. M., Spear D., Marais E., Kleynhans E. J., Symes A., Chanson J. \& Hoffmann M. 2010. Global indicators of biological invasion: species numbers, biodiversity impact and policy responses. $\mathrm{Di}$ versity \& Distrib. 16: 95-108.

Nentwig W. (ed.) 2007. Biological Invasions. Ecol. Stud. 193: $1-441$.

Niemivuo-Lahti J. (ed.) 2012. Finland's National Strategy on Invasive Alien Species. Ministry of Agriculture and Forestry, Finland. http://mmm.fi/documents/1410837/1894125/Finlands_national_strategy_on_invasive_alien_species.pdf. 
VON NUMERS M. 2011. Sea shore plants of the SW archipelago of Finland - distribution patterns and long term changes during the $20^{\text {th }}$ century. Ann. Bot. Fenn. 48(Suppl. A): $1-46$.

Olsoni B. 1946. Botaniskt från Ab Kimito-Hitis 1939-44. Memoranda Soc. Fauna Fl. Fenn. 21: 193-196.

ÖöPIK M., KuKK T., Kull K. \& Kull T. 2008. The importance of human mediation in species establishment: analysis of the alien flora of Estonia. Boreal Env. Res. 13: 53-67.

Pirinen P., Simola H., Aalto J., Kaukoranta J.-P., KarlsSON P. \& RuUhela R. 2012. Tilastoja Suomen ilmastosta 1981-2010. Climatological statistics of Finland 1981-2010. Finnish Meteorological Institute, Reports 1(2012): 1-83.

Rassi P., Hyvärinen E., Juslén A. \& Mannerkoski I. (eds) 2010. The 2010 Red list of Finnish species. Ministry of the Environment \& Finnish Environment Institute, Helsinki.

Raunio A., Schulman A. \& Kontula T. (eds) 2008. Assessment of threatened habitat types in Finland. Part 1: Results and basis for assessment \& Part 2: Habitat type descriptions. Suomen Ympäristö 8: 1-264 (in Finnish with English summary).

REDDERSEN J. 2006. Effekter på floraen ved tilgroning af beskyttede strandoverdrev med rynket rose (Rosa rugosa). Fl. \& Fauna (Esbjerg) 112: 75-84 (in Danish with English summary).

Ryttäri T., Kanerva T., Rintanen T., TAinio E. \& Teeriaho J. 2006. Present state of threatened seashore plants Salsola kali and Polygonum oxyspermum in the eastern archipelago of the Gulf of Finland. Lutukka 22: 67-82 (in Finnish with English summary).

Ryttäri T., Heiskala K., KeKäläInen H., Koskela K., voN Numers M., Rinkineva-Kantola L. \& Syrjänen K. 2014. Management of sandy seashores and dunes of Baltic Sea. Finnish Environment Institute, Helsinki (in Finnish and Swedish with English summary).

Scalera R., Genovesi P., Essl F. \& Rabitsch W. 2012. The impacts of invasive alien species in Europe. EEA Technical Report 16: 1-114.

Skult H. 1960. Om kärlväxtfloran i Korpo, Brunskär, en utskärsarkipelag. 1960. Acta Soc. Fauna Fl. Fenn. 76: 1-101.

SKYTÉN R. 1978. Sand- och dynsträndernas vegetation dess nedsliting. Nordenskiöld-samfundets Tidskrift 38: 37-49.
StJernberg T., Lindgren L., Cygnel M. 1974. Naturinventering inom glesbygden i Dragsfjärd. Dragsfjärds kommun, Helsingfors.

SYRJÄNEN K. 1995. Prickly saltwort (Salsola kali subsp. kali) on Jurmo island, Korppoo. Metsähallituksen Luonnonsuojelujulkaisuja, Sarja A 51: 1-49 (in Finnish with English summary).

Thiele J., Kollmann J. \& Andersen U. R. 2009. Ecological and socioeconomic correlates of plant invasions in Denmark: the utility of environmental assessment data. Ambio 38: 89-94.

Thiele J., Isermann M., Otte A. \& Kollmann J. 2010. Competitive displacement or biotic resistance? Disentangling relationships between community diversity and invasion success of tall herbs and shrubs. J. Veg. Sci. 21: 213-220.

Thiele J., Isermann M., Kollmann J. \& Otte A. 2011. Impact scores of invasive plants are biased by disregard of environmental co-variation and non-linearity. NeoBiota 10: 65-79.

Tyler T., Karlsson T., Milberg P., Sahlin U. \& Sundberg S. 2015. Invasive plant species in the Swedish flora: developing criteria and definitions, and assessing the invasiveness of individual taxa. Nordic J. Bot. 33: 300-317.

TzVelev N. N. (ed.) 2007. Environment and biological diversity of Berezovye Islands Archipelago (The Gulf of Finland). Committee on Natural Resources and Environmental Protection of the Leningrad Region Government, St. Petersburg (in Russian with English summary).

Veitch C. R., Clout M. N. \& Towns D. R. (eds) 2011. Island invasives: Eradication and management. Proceedings of the International Conference on Island Invasives. IUCN, Gland, Switzerland and CBB, Auckland, New Zealand.

Walker B. \& StefFen W. 1997. An overview of the implications of global change for natural and managed terrestrial ecosystems. Conservation Ecol. 1(2): 2.

WeIDEMA I. 2006. NOBANIS - Invasive Alien Species Fact Sheet - Rosa rugosa. Online Database of the European Network on Invasive Alien Species - NOBANIS. [14 Dec. 2016]. http://www.nobanis.org.

Weidema I., Ravn H. P., VestergaArd P., Johnsen I. \& Svart H. E. (eds) 2007. Rosa rugosa in Denmark Report. Biologisk Institut, Københavns Universitet, Skov- og Landskab, Københavns Universitet, samt Skov- og Naturstyrelsen. (in Danish with English summary). 
APPENDIX. Stands of Rosa rugosa Thunb. in Archipelago Sea National Park and its surroundings, recorded mainly in $2010-2016$. $\mathrm{NP}$ - national park, PNR - private nature reserve.

\begin{tabular}{|c|c|c|c|c|c|c|}
\hline Locality & Site & Coordinates & $\begin{array}{c}\text { Conservation } \\
\text { area }\end{array}$ & $\begin{array}{l}\text { Size } \\
{\left[\mathrm{m}^{2}\right]}\end{array}$ & Main habitat type & Note \\
\hline Utö & Halfaskärs kläppen & $\begin{array}{l}59^{\circ} 48^{\prime} 41.565^{\prime \prime} \mathrm{N} \\
21^{\circ} 24^{\prime} 32.305^{\prime \prime} \mathrm{E}\end{array}$ & - & & & Not found 2016 \\
\hline Utö & Slevharu & $\begin{array}{l}59^{\circ} 49^{\prime} 47.047^{\prime \prime} \mathrm{N} \\
21^{\circ} 18^{\prime} 27.742^{\prime \prime} \mathrm{E}\end{array}$ & - & 19 & Rock outcrop & \\
\hline Utö & Syndaskär & $\begin{array}{l}59^{\circ} 49^{\prime} 43.312^{\prime \prime} \mathrm{N} \\
21^{\circ} 25^{\prime} 43.696^{\prime \prime} \mathrm{E}\end{array}$ & - & 18 & $\begin{array}{l}\text { Seashore meadow } \\
\text { or dry meadow }\end{array}$ & \\
\hline Utö & Syndaskär & $\begin{array}{l}59^{\circ} 49^{\prime} 42.887^{\prime \prime} \mathrm{N} \\
21^{\circ} 25^{\prime} 41.322^{\prime \prime} \mathrm{E}\end{array}$ & - & 11 & Rock outcrop & \\
\hline Utö & Bokulla & $\begin{array}{l}59^{\circ} 50^{\prime} 1.946^{\prime \prime} \mathrm{N} \\
21^{\circ} 25^{\prime} 34.011^{\prime \prime} \mathrm{E}\end{array}$ & NP & 14 & Rock outcrop & \\
\hline Utö & Huslandet (Bokulla) & $\begin{array}{l}59^{\circ} 50^{\prime} 17.909^{\prime \prime} \mathrm{N} \\
21^{\circ} 25^{\prime} 38.478^{\prime \prime} \mathrm{E}\end{array}$ & NP & 2 & $\begin{array}{l}\text { Seashore meadow } \\
\text { or dry meadow }\end{array}$ & \\
\hline Utö & Utö, Kesnäs bukten & $\begin{array}{l}59^{\circ} 46^{\prime} 42.789^{\prime \prime} \mathrm{N} \\
21^{\circ} 22^{\prime} 17.031^{\prime \prime} \mathrm{E}\end{array}$ & NP & 17 & $\begin{array}{l}\text { Seashore meadow } \\
\text { or dry meadow }\end{array}$ & \\
\hline Utö & Utö, Kesnäs & $\begin{array}{l}59^{\circ} 46^{\prime} 33.423^{\prime \prime} \mathrm{N} \\
21^{\circ} 22^{\prime} 21.439^{\prime \prime} \mathrm{E}\end{array}$ & NP & 4 & Stony shore & \\
\hline Utö & Utö, S-shore & $\begin{array}{l}59^{\circ} 46^{\prime} 35.669^{\prime \prime} \mathrm{N} \\
21^{\circ} 22^{\prime} 46.626^{\prime \prime} \mathrm{E}\end{array}$ & NP & 10 & Stony shore & \\
\hline Utö & Utö, SE-shore & $\begin{array}{l}59^{\circ} 46^{\prime} 39.305^{\prime \prime} \mathrm{N} \\
21^{\circ} 22^{\prime} 57.383^{\prime \prime} \mathrm{E}\end{array}$ & NP & 3 & Stony shore & \\
\hline Utö & Utö, Enskär & $\begin{array}{l}59^{\circ} 47^{\prime} 1.268^{\prime \prime} \mathrm{N} \\
21^{\circ} 21^{\prime} 51.036^{\prime \prime} \mathrm{E}\end{array}$ & - & 12 & Stony shore & \\
\hline Utö & Utö, Enskär & $\begin{array}{l}59^{\circ} 47^{\prime} 0.878^{\prime \prime} \mathrm{N}, \\
21^{\circ} 21^{\prime} 51.744^{\prime \prime} \mathrm{E}\end{array}$ & - & 1 & Stony shore & \\
\hline Utö & Utö, NE-shore & $\begin{array}{l}59^{\circ} 46^{\prime} 57.334^{\prime \prime} \mathrm{N} \\
21^{\circ} 22^{\prime} 8.02^{\prime \prime} \mathrm{E}\end{array}$ & - & 6 & $\begin{array}{l}\text { Seashore meadow } \\
\text { or dry meadow }\end{array}$ & \\
\hline Utö & Trutgrund & $\begin{array}{l}59^{\circ} 46^{\prime} 45.497^{\prime \prime} \mathrm{N} \\
21^{\circ} 21^{\prime} 39.056^{\prime \prime} \mathrm{E}\end{array}$ & - & 6 & Stony shore & \\
\hline Utö & Gråkobbarna & $\begin{array}{l}59^{\circ} 47^{\prime} 11.556^{\prime \prime} \mathrm{N} \\
21^{\circ} 23^{\prime} 45.599^{\prime \prime} \mathrm{E}\end{array}$ & - & 2 & Stony shore & \\
\hline Utö & Menfolskär & $\begin{array}{l}59^{\circ} 47^{\prime} 15.008^{\prime \prime} \mathrm{N} \\
21^{\circ} 23^{\prime} 20.279^{\prime \prime} \mathrm{E}\end{array}$ & NP & 1 & Rock outcrop & \\
\hline Utö & Gommaskär & $\begin{array}{l}59^{\circ} 47^{\prime} 19.275^{\prime \prime} \mathrm{N} \\
21^{\circ} 23^{\prime} 24.637^{\prime \prime} \mathrm{E}\end{array}$ & NP & 1 & $\begin{array}{l}\text { Seashore meadow } \\
\text { or dry meadow }\end{array}$ & \\
\hline Utö & Gommaskär & $\begin{array}{l}59^{\circ} 47^{\prime} 19.275^{\prime \prime} \mathrm{N} \\
21^{\circ} 23^{\prime} 24.637^{\prime \prime} \mathrm{E}\end{array}$ & NP & 46 & $\begin{array}{l}\text { Seashore meadow } \\
\text { or dry meadow }\end{array}$ & \\
\hline Utö & Gommaskär & $\begin{array}{l}59^{\circ} 47^{\prime} 16.752^{\prime \prime} \mathrm{N} \\
21^{\circ} 23^{\prime} 19.407^{\prime \prime} \mathrm{E}\end{array}$ & NP & 64 & $\begin{array}{l}\text { Seashore meadow } \\
\text { or dry meadow }\end{array}$ & \\
\hline Utö & Gommaskär & $\begin{array}{l}59^{\circ} 47^{\prime} 18.343^{\prime \prime} \mathrm{N} \\
21^{\circ} 23^{\prime} 31.606^{\prime \prime} \mathrm{E}\end{array}$ & NP & 1 & Stony shore & \\
\hline Utö & Stenskär & $\begin{array}{l}59^{\circ} 47^{\prime} 10.984^{\prime \prime} \mathrm{N} \\
21^{\circ} 23^{\prime} 9.519^{\prime \prime} \mathrm{E}\end{array}$ & NP & 5 & Stony shore & \\
\hline Utö & Revskär & $\begin{array}{l}59^{\circ} 47^{\prime} 33.661^{\prime \prime} \mathrm{N} \\
21^{\circ} 23^{\prime} 12.638^{\prime \prime} \mathrm{E}\end{array}$ & NP & 1 & $\begin{array}{l}\text { Seashore meadow } \\
\text { or dry meadow }\end{array}$ & \\
\hline Utö & Sundskär & $\begin{array}{l}59^{\circ} 47^{\prime} 8.848^{\prime \prime} \mathrm{N} \\
21^{\circ} 24^{\prime} 22.294^{\prime \prime} \mathrm{E}\end{array}$ & NP & 8 & Sandy beach & \\
\hline Utö & Sundskär & $\begin{array}{l}59^{\circ} 47^{\prime} 12.337^{\prime \prime} \mathrm{N} \\
21^{\circ} 24^{\prime} 25.884^{\prime \prime} \mathrm{E}\end{array}$ & NP & 18 & $\begin{array}{l}\text { Seashore meadow } \\
\text { or dry meadow }\end{array}$ & \\
\hline Utö & Ormskär & $\begin{array}{l}59^{\circ} 47^{\prime} 35.55^{\prime \prime} \mathrm{N}, \\
21^{\circ} 22^{\prime} 21.483^{\prime \prime} \mathrm{E}\end{array}$ & NP & 7 & $\begin{array}{l}\text { Seashore meadow } \\
\text { or dry meadow }\end{array}$ & \\
\hline Utö & Ormskär & $\begin{array}{l}59^{\circ} 47^{\prime} 31.673^{\prime \prime} \mathrm{N}, \\
21^{\circ} 22^{\prime} 8.127^{\prime \prime} \mathrm{E}\end{array}$ & NP & 3 & Stony shore & \\
\hline
\end{tabular}


ApPENDIX. Continued.

\begin{tabular}{|c|c|c|c|c|c|c|}
\hline Locality & Site & Coordinates & $\begin{array}{c}\text { Conservation } \\
\text { area }\end{array}$ & $\begin{array}{l}\text { Size } \\
{\left[\mathrm{m}^{2}\right]}\end{array}$ & Main habitat type & Note \\
\hline Utö & Ormskär & $\begin{array}{l}59^{\circ} 47^{\prime} 21.205^{\prime \prime} \mathrm{N} \\
21^{\circ} 22^{\prime} 20.822^{\prime \prime} \mathrm{E}\end{array}$ & NP & 2 & Stony shore & \\
\hline Utö & Ormskär & $\begin{array}{l}59^{\circ} 47^{\prime} 12.173^{\prime \prime} \mathrm{N}, \\
21^{\circ} 22^{\prime} 30.765^{\prime \prime} \mathrm{E}\end{array}$ & NP & 5 & Stony shore & \\
\hline Utö & Örskär & $\begin{array}{l}59^{\circ} 48^{\prime} 36.354^{\prime \prime} \mathrm{N}, \\
21^{\circ} 27^{\prime} 40.483^{\prime \prime} \mathrm{E}\end{array}$ & NP & 4 & Sandy beach & \\
\hline Utö & Örskärs ören & $\begin{array}{l}59^{\circ} 48^{\prime} 57.31^{\prime \prime} \mathrm{N} \\
21^{\circ} 27^{\prime} 58.924^{\prime \prime} \mathrm{E}\end{array}$ & NP & 304 & Sandy beach & Also sandy meadow \\
\hline Utö & Örskärs ören & $\begin{array}{l}59^{\circ} 48^{\prime} 56.323^{\prime \prime} \mathrm{N}, \\
21^{\circ} 27^{\prime} 54.715^{\prime \prime} \mathrm{E}\end{array}$ & NP & 2 & Sandy beach & Also sandy meadow \\
\hline Utö & Örskärs ören & $\begin{array}{l}59^{\circ} 48^{\prime} 55.054^{\prime \prime} \mathrm{N}, \\
21^{\circ} 27^{\prime} 48.431^{\prime \prime} \mathrm{E}\end{array}$ & NP & 88 & Sandy beach & Also sandy meadow \\
\hline Jurmo & Jurmo, harbour & $\begin{array}{l}59^{\circ} 49^{\prime} 35.321^{\prime} \mathrm{N}^{\prime}, \\
21^{\circ} 35^{\prime} 7.958^{\prime \prime} \mathrm{E}\end{array}$ & - & 0.5 & Stony shore & \\
\hline Jurmo & Estrevlarna & $\begin{array}{l}59^{\circ} 48^{\prime} 50.353^{\prime \prime} \mathrm{N} \\
21^{\circ} 33^{\prime} 35.16^{\prime \prime} \mathrm{E} \\
\end{array}$ & NP & 100 & Sandy beach & $\begin{array}{l}\text { Control since } 2010 \text {, } \\
\text { covered }\end{array}$ \\
\hline Jurmo & Estrevlarna & $\begin{array}{l}59^{\circ} 48^{\prime} 50.353^{\prime \prime} \mathrm{N}, \\
21^{\circ} 33^{\prime} 35.16^{\prime \prime} \mathrm{E}\end{array}$ & NP & 70 & Sandy beach & $\begin{array}{l}\text { Control since } 2010 \text {, } \\
\text { covered }\end{array}$ \\
\hline Jurmo & Estrevlarna & $\begin{array}{l}59^{\circ} 49^{\prime} 1.789^{\prime \prime} \mathrm{N} \\
21^{\circ} 34^{\prime} 25.905^{\prime \prime} \mathrm{E}\end{array}$ & NP & 1 & Sandy beach & Uprooted 2016 \\
\hline Jurmo & Jurmo, Västerrevet & $\begin{array}{l}59^{\circ} 48^{\prime} 56.886^{\prime \prime} \mathrm{N}, \\
21^{\circ} 33^{\prime} 45.088^{\prime \prime} \mathrm{E}\end{array}$ & NP & 0.5 & Sandy beach & Uprooted 2014 \\
\hline Jurmo & Jurmo, Lägnörs fladan & $\begin{array}{l}59^{\circ} 49^{\prime} 9.289^{\prime \prime} \mathrm{N} \\
21^{\circ} 34^{\prime} 30.215^{\prime \prime} \mathrm{E}\end{array}$ & NP & 0.5 & Sandy beach & Uprooted 2013 \\
\hline Jurmo & Jurmo, Tvåstenviken & $\begin{array}{l}59^{\circ} 49^{\prime} 12.562^{\prime \prime} \mathrm{N}, \\
21^{\circ} 35^{\prime} 13.387^{\prime \prime} \mathrm{E}\end{array}$ & NP & 1 & Sandy beach & Uprooted 2010 \\
\hline Jurmo & Jurmo, Tvåstenviken & $\begin{array}{l}59^{\circ} 49^{\prime} 11.202^{\prime \prime} \mathrm{N}, \\
21^{\circ} 35^{\prime} 10.578^{\prime \prime} \mathrm{E}\end{array}$ & NP & 0.5 & Stony shore & \\
\hline Jurmo & Jurmo, Estnäs & $\begin{array}{l}59^{\circ} 49^{\prime} 29.808^{\prime \prime} \mathrm{N}, \\
21^{\circ} 37^{\prime} 16.568^{\prime \prime} \mathrm{E}\end{array}$ & NP & 1 & Dwarf shrub heath & $\begin{array}{l}\text { Uprooted } 2010 \text { and } \\
2013, \text { covered }\end{array}$ \\
\hline Jurmo & Jurmo, Estnäs & $\begin{array}{l}59^{\circ} 49^{\prime} 27.699^{\prime \prime} \mathrm{N}, \\
21^{\circ} 37^{\prime} 25.188^{\prime \prime} \mathrm{E}\end{array}$ & NP & 1 & Stony shore & \\
\hline Jurmo & Jurmo, Estnäs & $\begin{array}{l}59^{\circ} 49^{\prime} 31.931^{\prime \prime} \mathrm{N}, \\
21^{\circ} 37^{\prime} 0.396^{\prime \prime} \mathrm{E}\end{array}$ & PNR & 1 & Stony shore & Uprooted 2015 \\
\hline Jurmo & Sorgen & $\begin{array}{l}59^{\circ} 49^{\prime} 28.222^{\prime \prime} \mathrm{N}, \\
21^{\circ} 36^{\prime} 17.414^{\prime \prime} \mathrm{E}\end{array}$ & NP & 2 & Sandy beach & Eradicated \\
\hline Jurmo & Jurmo, Österrevet & $\begin{array}{l}59^{\circ} 50^{\prime} 0.777^{\prime \prime} \mathrm{N}, \\
21^{\circ} 38^{\prime} 13.517^{\prime \prime} \mathrm{E}\end{array}$ & NP & 0.5 & Stony shore & Eradicated \\
\hline Jurmo & $\begin{array}{l}\text { Sorgen (Sorgenkläp- } \\
\text { parna) }\end{array}$ & $\begin{array}{l}59^{\circ} 49^{\prime} 21.047^{\prime \prime} \mathrm{N}, \\
21^{\circ} 36^{\prime} 21.283^{\prime \prime} \mathrm{E}\end{array}$ & - & 11 & Stony shore & \\
\hline Jurmo & Sanden & $\begin{array}{l}59^{\circ} 50^{\prime} 24.995^{\prime \prime} \mathrm{N}, \\
21^{\circ} 39^{\prime} 33.474^{\prime \prime} \mathrm{E}\end{array}$ & NP & 35 & Sandy beach & Control started \\
\hline Jurmo & Grundvik harun & $\begin{array}{l}59^{\circ} 49^{\prime} 26.011^{\prime \prime} \mathrm{N}, \\
21^{\circ} 37^{\prime} 52.322^{\prime \prime} \mathrm{E}\end{array}$ & - & 44 & $\begin{array}{l}\text { Seashore meadow } \\
\text { or dry meadow }\end{array}$ & \\
\hline Jurmo & Killingharu & $\begin{array}{l}59^{\circ} 49^{\prime} 31.953^{\prime \prime} \mathrm{N}, \\
21^{\circ} 32^{\prime} 41.723^{\prime \prime} \mathrm{E}\end{array}$ & NP & 1 & Stony shore & \\
\hline Jurmo & Gåsharu & $\begin{array}{l}59^{\circ} 48^{\prime} 28.696^{\prime \prime} \mathrm{N}, \\
21^{\circ} 40^{\prime} 7.431^{\prime \prime} \mathrm{E}\end{array}$ & PNR & 23 & $\begin{array}{l}\text { Seashore meadow } \\
\text { or dry meadow }\end{array}$ & \\
\hline Jurmo & Lökharu & $\begin{array}{l}59^{\circ} 49^{\prime} 6.332^{\prime \prime} \mathrm{N}, \\
21^{\circ} 42^{\prime} 31.611^{\prime \prime} \mathrm{E}\end{array}$ & NP & 9 & Stony shore & \\
\hline Jurmo & Stora Örskär & $\begin{array}{l}59^{\circ} 50^{\prime} 22.686^{\prime \prime} \mathrm{N}, \\
21^{\circ} 41^{\prime} 43.432^{\prime \prime} \mathrm{E}\end{array}$ & PNR & 24 & Stony shore & \\
\hline Jurmo & Stora Örskär & $\begin{array}{l}59^{\circ} 50^{\prime} 23.248^{\prime} \mathrm{N}^{\prime} \\
21^{\circ} 41^{\prime} 42.12^{\prime \prime} \mathrm{E} \\
\end{array}$ & PNR & 4 & Stony shore & \\
\hline
\end{tabular}


ApPENDIX. Continued.

\begin{tabular}{|c|c|c|c|c|c|c|}
\hline Locality & Site & Coordinates & $\begin{array}{c}\text { Conservation } \\
\text { area }\end{array}$ & $\begin{array}{l}\text { Size } \\
{\left[\mathrm{m}^{2}\right]}\end{array}$ & Main habitat type & Note \\
\hline Jurmo & Stora Örskär & $\begin{array}{l}59^{\circ} 50^{\prime} 21.02^{\prime \prime} \mathrm{N} \\
21^{\circ} 41^{\prime} 47.88^{\prime \prime} \mathrm{E}\end{array}$ & PNR & 33 & $\begin{array}{l}\text { Seashore meadow } \\
\text { or dry meadow }\end{array}$ & \\
\hline Jurmo & Stora Örskär & $\begin{array}{l}59^{\circ} 50^{\prime} 18.481^{\prime \prime} \mathrm{N}, \\
21^{\circ} 41^{\prime} 58.772^{\prime \prime} \mathrm{E}\end{array}$ & PNR & 3 & $\begin{array}{l}\text { Seashore meadow } \\
\text { or dry meadow }\end{array}$ & \\
\hline Jurmo & Lilla Örskär & $\begin{array}{l}59^{\circ} 50^{\prime} 4.103^{\prime \prime} \mathrm{N} \\
21^{\circ} 41^{\prime} 9.919^{\prime \prime} \mathrm{E}\end{array}$ & PNR & 42 & $\begin{array}{l}\text { Seashore meadow } \\
\text { or dry meadow }\end{array}$ & \\
\hline Jurmo & Lilla Örskär & $\begin{array}{l}59^{\circ} 50^{\prime} 4.11^{\prime \prime} \mathrm{N}, \\
21^{\circ} 41^{\prime} 12.491^{\prime \prime} \mathrm{E}\end{array}$ & PNR & 14 & Stony shore & \\
\hline Jurmo & Lilla Örskär & $\begin{array}{l}59^{\circ} 50^{\prime} 4.846^{\prime \prime} \mathrm{N}, \\
21^{\circ} 41^{\prime} 13.854^{\prime \prime} \mathrm{E}\end{array}$ & PNR & 65 & $\begin{array}{l}\text { Seashore meadow } \\
\text { or dry meadow }\end{array}$ & \\
\hline Jurmo & Lilla Örskär & $\begin{array}{l}59^{\circ} 50^{\prime} 4.547^{\prime \prime} \mathrm{N}, \\
21^{\circ} 41^{\prime} 7.275^{\prime \prime} \mathrm{E}\end{array}$ & PNR & 15 & Stony shore & \\
\hline Jurmo & Örskärs ören & $\begin{array}{l}59^{\circ} 49^{\prime} 56.964^{\prime \prime} \mathrm{N}, \\
21^{\circ} 41^{\prime} 47.984^{\prime \prime} \mathrm{E}\end{array}$ & PNR & 48 & $\begin{array}{l}\text { Seashore meadow } \\
\text { or dry meadow }\end{array}$ & \\
\hline Jurmo & Huslandet (Gaddarna) & $\begin{array}{l}59^{\circ} 47^{\prime} 20.137^{\prime \prime} \mathrm{N}, \\
21^{\circ} 30^{\prime} 44.396^{\prime \prime} \mathrm{E}\end{array}$ & NP & 2 & Rock outcrop & \\
\hline Jurmo & Norrkläpparna & $\begin{array}{l}59^{\circ} 50^{\prime} 19.106^{\prime \prime} \mathrm{N}, \\
21^{\circ} 36^{\prime} 40.049^{\prime \prime} \mathrm{E}\end{array}$ & PNR & 11 & Stony shore & \\
\hline Jurmo & Norrkläpparna & $\begin{array}{l}59^{\circ} 50^{\prime} 18.173^{\prime \prime} \mathrm{N}, \\
21^{\circ} 36^{\prime} 40.2^{\prime \prime} \mathrm{E}\end{array}$ & PNR & 1 & $\begin{array}{l}\text { Seashore meadow } \\
\text { or dry meadow }\end{array}$ & \\
\hline Jurmo & Norrkläpparna & $\begin{array}{l}59^{\circ} 50^{\prime} 18.223^{\prime \prime} \mathrm{N}, \\
21^{\circ} 36^{\prime} 40.642^{\prime \prime} \mathrm{E}\end{array}$ & PNR & 2 & $\begin{array}{l}\text { Seashore meadow } \\
\text { or dry meadow }\end{array}$ & \\
\hline Trunsö & Glasaskär & $\begin{array}{l}59^{\circ} 49^{\prime} 14.603^{\prime \prime} \mathrm{N}, \\
21^{\circ} 47^{\prime} 29.047^{\prime \prime} \mathrm{E}\end{array}$ & NP & 2 & Stony shore & \\
\hline Trunsö & Glasaskär & $\begin{array}{l}59^{\circ} 49^{\prime} 15.135^{\prime \prime} \mathrm{N}, \\
21^{\circ} 47^{\prime} 31.022^{\prime \prime} \mathrm{E}\end{array}$ & NP & 15 & Stony shore & \\
\hline Trunsö & Lökskär SE & $\begin{array}{l}59^{\circ} 49^{\prime} 15.849^{\prime \prime} \mathrm{N}, \\
21^{\circ} 49^{\prime} 6.782^{\prime \prime} \mathrm{E}\end{array}$ & NP & 1 & Stony shore & \\
\hline Trunsö & Lökskär SE & $\begin{array}{l}59^{\circ} 49^{\prime} 15.873^{\prime \prime} \mathrm{N}, \\
21^{\circ} 49^{\prime} 6.586^{\prime \prime} \mathrm{E}\end{array}$ & NP & 2 & Stony shore & \\
\hline Trunsö & Lökskär SE & $\begin{array}{l}59^{\circ} 49^{\prime} 16.069^{\prime \prime} \mathrm{N}, \\
21^{\circ} 49^{\prime} 6.62^{\prime \prime} \mathrm{E}\end{array}$ & NP & 1 & Stony shore & \\
\hline Trunsö & Långören (southern) & $\begin{array}{l}59^{\circ} 48^{\prime} 39.399^{\prime \prime} \mathrm{N}, \\
21^{\circ} 50^{\prime} 47.332^{\prime \prime} \mathrm{E}\end{array}$ & NP & 3 & Stony shore & \\
\hline Trunsö & Långören (southern) & $\begin{array}{l}59^{\circ} 48^{\prime} 34.365^{\prime \prime} \mathrm{N}, \\
21^{\circ} 50^{\prime} 45.283^{\prime \prime} \mathrm{E}\end{array}$ & NP & 27 & Stony shore & \\
\hline Trunsö & Kistskär & $\begin{array}{l}59^{\circ} 52^{\prime} 7.866^{\prime \prime} \mathrm{N}, \\
21^{\circ} 46^{\prime} 49.909^{\prime \prime} \mathrm{E}\end{array}$ & NP & 9 & $\begin{array}{l}\text { Seashore meadow } \\
\text { or dry meadow }\end{array}$ & $\begin{array}{l}\text { Uprooted } 2014 \text { and } \\
2016 \text {, greatly reduced }\end{array}$ \\
\hline Trunsö & Kistskär & $\begin{array}{l}59^{\circ} 52^{\prime} 10.352^{\prime \prime} \mathrm{N}, \\
21^{\circ} 47^{\prime} 4.393^{\prime \prime} \mathrm{E}\end{array}$ & NP & 1 & Stony shore & Uprooted 2016 \\
\hline Trunsö & Kvessören & $\begin{array}{l}59^{\circ} 53^{\prime} 30.828^{\prime \prime} \mathrm{N}, \\
21^{\circ} 50^{\prime} 10.861^{\prime \prime} \mathrm{E}\end{array}$ & - & 58 & Stony shore & \\
\hline Trunsö & Kläpparna NW & $\begin{array}{l}59^{\circ} 53^{\prime} 31.531^{\prime \prime} \mathrm{N}, \\
21^{\circ} 50^{\prime} 41.165^{\prime \prime} \mathrm{E}\end{array}$ & - & 12 & Stony shore & \\
\hline Trunsö & Vitskären S-island & $\begin{array}{l}59^{\circ} 53^{\prime} 16.025^{\prime \prime} \mathrm{N}, \\
21^{\circ} 50^{\prime} 53.624^{\prime \prime} \mathrm{E}\end{array}$ & - & 12 & Stony shore & \\
\hline Trunsö & Vitskären S-island & $\begin{array}{l}59^{\circ} 53^{\prime} 16.001^{\prime \prime} \mathrm{N}, \\
21^{\circ} 50^{\prime} 53.821^{\prime \prime} \mathrm{E}\end{array}$ & - & 2 & Stony shore & \\
\hline Trunsö & Vitskären S-island & $\begin{array}{l}59^{\circ} 53^{\prime} 17.361^{\prime \prime} \mathrm{N}, \\
21^{\circ} 50^{\prime} 46.394^{\prime \prime} \mathrm{E}\end{array}$ & - & 4 & $\begin{array}{l}\text { Seashore meadow } \\
\text { or dry meadow }\end{array}$ & \\
\hline Trunsö & Vitskär & $\begin{array}{l}59^{\circ} 53^{\prime} 19.461^{\prime \prime} \mathrm{N}, \\
21^{\circ} 47^{\prime} 41.409^{\prime \prime} \mathrm{E}\end{array}$ & - & 6 & Stony shore & $\begin{array}{l}\text { Grew partly under } \\
\text { black alders }\end{array}$ \\
\hline Trunsö & Långören (western) & $\begin{array}{l}59^{\circ} 52^{\prime} 35.223^{\prime \prime} \mathrm{N}, \\
21^{\circ} 45^{\prime} 17.109^{\prime \prime} \mathrm{E}\end{array}$ & - & 8 & Sandy beach & $\begin{array}{l}\text { Uprooted } 2014 \text { and } \\
2016 \text {, greatly reduced }\end{array}$ \\
\hline
\end{tabular}


ApPENDIX. Continued.

\begin{tabular}{|c|c|c|c|c|c|c|}
\hline Locality & Site & Coordinates & \begin{tabular}{|c|}
$\begin{array}{c}\text { Conservation } \\
\text { area }\end{array}$ \\
\end{tabular} & $\begin{array}{l}\text { Size } \\
{\left[\mathrm{m}^{2}\right]}\end{array}$ & Main habitat type & Note \\
\hline Trunsö & Garpharu E-skerry & $\begin{array}{l}59^{\circ} 48^{\prime} 42.599^{\prime \prime} \mathrm{N} \\
21^{\circ} 49^{\prime} 19.992^{\prime \prime} \mathrm{E}\end{array}$ & NP & 25 & Stony shore & \\
\hline Trunsö & Ytterören & $\begin{array}{l}59^{\circ} 50^{\prime} 20.003^{\prime \prime} \mathrm{N} \\
21^{\circ} 46^{\prime} 57.896^{\prime \prime} \mathrm{E}\end{array}$ & - & 34 & Stony shore & \\
\hline Trunsö & Salskärs pattrorna & $\begin{array}{l}59^{\circ} 50^{\prime} 47.765^{\prime \prime} \mathrm{N} \\
21^{\circ} 50^{\prime} 56.074^{\prime \prime} \mathrm{E}\end{array}$ & - & 4 & Stony shore & \\
\hline Trunsö & Stora Salskär & $\begin{array}{l}59^{\circ} 50^{\prime} 46.716^{\prime \prime} \mathrm{N} \\
21^{\circ} 50^{\prime} 16.148^{\prime \prime} \mathrm{E}\end{array}$ & NP & 16 & Stony shore & \\
\hline Trunsö & Stora Salskär & $\begin{array}{l}59^{\circ} 50^{\prime} 43.022^{\prime \prime} \mathrm{N} \\
21^{\circ} 50^{\prime} 16.078^{\prime \prime} \mathrm{E}\end{array}$ & NP & 3 & Stony shore & \\
\hline Trunsö & Stora Salskär & $\begin{array}{l}59^{\circ} 50^{\prime} 52.172^{\prime \prime} \mathrm{N} \\
21^{\circ} 50^{\prime} 16.588^{\prime \prime} \mathrm{E}\end{array}$ & NP & 4 & Stony shore & \\
\hline Trunsö & Alskär & $\begin{array}{l}59^{\circ} 48^{\prime} 58.872^{\prime \prime} \mathrm{N}, \\
21^{\circ} 48^{\prime} 46.023^{\prime \prime} \mathrm{E}\end{array}$ & NP & 15 & $\begin{array}{l}\text { Seashore meadow } \\
\text { or dry meadow }\end{array}$ & \\
\hline Trunsö & Grabbaskläppen & $\begin{array}{l}59^{\circ} 49^{\prime} 7.254^{\prime \prime} \mathrm{N} \\
21^{\circ} 49^{\prime} 4.777^{\prime \prime} \mathrm{E}\end{array}$ & NP & 20 & Stony shore & \\
\hline Trunsö & $\begin{array}{l}\text { Kummelskären S- } \\
\text { skerry }\end{array}$ & $\begin{array}{l}59^{\circ} 49^{\prime} 46.509^{\prime \prime} \mathrm{N} \\
21^{\circ} 50^{\prime} 54.496^{\prime \prime} \mathrm{E}\end{array}$ & NP & 4 & Stony shore & \\
\hline Trunsö & Långskär & $\begin{array}{l}59^{\circ} 49^{\prime} 24.162^{\prime \prime} \mathrm{N} \\
21^{\circ} 47^{\prime} 58.611^{\prime \prime} \mathrm{E}\end{array}$ & NP & 5 & Stony shore & \\
\hline Trunsö & Harun & $\begin{array}{l}59^{\circ} 52^{\prime} 3.059^{\prime \prime} \mathrm{N} \\
21^{\circ} 45^{\prime} 3.064^{\prime \prime} \mathrm{E}\end{array}$ & - & 30 & Rock outcrop & \\
\hline Trunsö & Harun & $\begin{array}{l}59^{\circ} 52^{\prime} 1.75^{\prime \prime} \mathrm{N} \\
21^{\circ} 45^{\prime} 7.585^{\prime \prime} \mathrm{E}\end{array}$ & - & 5 & Stony shore & \\
\hline Trunsö & Stenkläppen & $\begin{array}{l}59^{\circ} 48^{\prime} 39.824^{\prime \prime} \mathrm{N}, \\
21^{\circ} 51^{\prime} 8.864^{\prime \prime} \mathrm{E}\end{array}$ & NP & 7 & Stony shore & \\
\hline Trunsö & Låga Sundskär & $\begin{array}{l}59^{\circ} 52^{\prime} 7.971^{\prime \prime} \mathrm{N} \\
21^{\circ} 48^{\prime} 28.157^{\prime \prime} \mathrm{E}\end{array}$ & NP & & & Not found 2014 \\
\hline Lökholm & Skogsskär & $\begin{array}{l}59^{\circ} 54^{\prime} 17.799^{\prime \prime} \mathrm{N} \\
21^{\circ} 51^{\prime} 15.875^{\prime \prime} \mathrm{E}\end{array}$ & - & 26 & Stony shore & \\
\hline Lökholm & Skogsskär & $\begin{array}{l}59^{\circ} 54^{\prime} 18.094^{\prime \prime} \mathrm{N} \\
21^{\circ} 51^{\prime} 26.723^{\prime \prime} \mathrm{E}\end{array}$ & - & 3 & Stony shore & $\begin{array}{l}\text { Grew under black } \\
\text { alders }\end{array}$ \\
\hline Lökholm & $\begin{array}{l}\text { Sten-Kummelskär, } \\
\text { N-skerry }\end{array}$ & $\begin{array}{l}59^{\circ} 54^{\prime} 12.111^{\prime \prime} \mathrm{N} \\
21^{\circ} 52^{\prime} 2.522^{\prime \prime} \mathrm{E}\end{array}$ & - & 2 & Stony shore & \\
\hline Österskär & Norrkläppen & $\begin{array}{l}59^{\circ} 58^{\prime} 9.94^{\prime \prime} \mathrm{N} \\
21^{\circ} 15^{\prime} 28.825^{\prime \prime} \mathrm{E}\end{array}$ & NP & 10 & Stony shore & \\
\hline Österskär & Bredskär, NE-skerry & $\begin{array}{l}59^{\circ} 58^{\prime} 4.128^{\prime \prime} \mathrm{N} \\
21^{\circ} 15^{\prime} 15.166^{\prime \prime} \mathrm{E}\end{array}$ & NP & 8 & Stony shore & \\
\hline Österskär & Bredskär & $\begin{array}{l}59^{\circ} 57^{\prime} 59.791^{\prime \prime} \mathrm{N} \\
21^{\circ} 15^{\prime} 14.498^{\prime \prime} \mathrm{E}\end{array}$ & NP & 0.5 & Rock outcrop & \\
\hline Österskär & Långharu, W-skerry & $\begin{array}{l}59^{\circ} 57^{\prime} 18.679^{\prime \prime} \mathrm{N} \\
21^{\circ} 14^{\prime} 32.937^{\prime \prime} \mathrm{E}\end{array}$ & NP & 13 & Stony shore & \\
\hline Österskär & Råkläpparna & $\begin{array}{l}59^{\circ} 56^{\prime} 7.632^{\prime \prime} \mathrm{N}, \\
21^{\circ} 20^{\prime} 36.964^{\prime \prime} \mathrm{E}\end{array}$ & NP & 27 & $\begin{array}{l}\text { Seashore meadow } \\
\text { or dry meadow }\end{array}$ & \\
\hline Österskär & Kummelkläpparna & $\begin{array}{l}59^{\circ} 56^{\prime} 7.896^{\prime \prime} \mathrm{N} \\
21^{\circ} 20^{\prime} 12.261^{\prime \prime} \mathrm{E}\end{array}$ & NP & 6 & $\begin{array}{l}\text { Seashore meadow } \\
\text { or dry meadow }\end{array}$ & \\
\hline Österskär & Värpeln & $\begin{array}{l}59^{\circ} 55^{\prime} 17.006^{\prime \prime} \mathrm{N} \\
21^{\circ} 21^{\prime} 9.415^{\prime \prime} \mathrm{E}\end{array}$ & NP & 7 & Stony shore & \\
\hline Brunskär & Långlandet & $\begin{array}{l}60^{\circ} 2^{\prime} 18.77^{\prime \prime} \mathrm{N}, \\
21^{\circ} 36^{\prime} 27.981^{\prime \prime} \mathrm{E}\end{array}$ & NP & 100 & $\begin{array}{l}\text { Seashore meadow } \\
\text { or dry meadow }\end{array}$ & \\
\hline Brunskär & $\begin{array}{l}\text { Lilla Rönnskär, S- } \\
\text { skerry }\end{array}$ & $\begin{array}{l}60^{\circ} 0^{\prime} 51.506^{\prime \prime} \mathrm{N} \\
21^{\circ} 29^{\prime} 21.756^{\prime \prime} \mathrm{E}\end{array}$ & NP & 11 & Stony shore & \\
\hline Brunskär & Rågskär, SE-skerry & $\begin{array}{l}60^{\circ} 2^{\prime} 19.521^{\prime \prime} \mathrm{N} \\
21^{\circ} 36^{\prime} 47.926^{\prime \prime} \mathrm{E}\end{array}$ & NP & 13 & $\begin{array}{l}\text { Seashore meadow } \\
\text { or dry meadow }\end{array}$ & \\
\hline
\end{tabular}


ApPENDIX. Continued.

\begin{tabular}{|c|c|c|c|c|c|c|}
\hline Locality & Site & Coordinates & \begin{tabular}{|c|}
$\begin{array}{c}\text { Conservation } \\
\text { area }\end{array}$ \\
\end{tabular} & $\begin{array}{l}\text { Size } \\
{\left[\mathrm{m}^{2}\right]}\end{array}$ & Main habitat type & Note \\
\hline Aspö & Snökobben & $\begin{array}{l}59^{\circ} 55^{\prime} 37.539^{\prime \prime} \mathrm{N}, \\
21^{\circ} 30^{\prime} 38.329^{\prime \prime} \mathrm{E}\end{array}$ & PNR & 0.5 & Rock outcrop & \\
\hline Aspö & Vidskär & $\begin{array}{l}59^{\circ} 54^{\prime} 35.262^{\prime \prime} \mathrm{N}, \\
59^{\circ} 54^{\prime} 35.262^{\prime \prime} \mathrm{E}\end{array}$ & PNR & 1 & Stony shore & $\begin{array}{l}\text { Grew partly under } \\
\text { black alders }\end{array}$ \\
\hline Aspö & Mossaskär & $\begin{array}{l}59^{\circ} 53^{\prime} 45.777^{\prime \prime} \mathrm{N}, \\
21^{\circ} 35^{\prime} 36.064^{\prime \prime} \mathrm{E}\end{array}$ & NP & 2 & $\begin{array}{l}\text { Seashore meadow } \\
\text { or dry meadow }\end{array}$ & \\
\hline Aspö & Mossaskär & $\begin{array}{l}59^{\circ} 53^{\prime} 41.411^{\prime \prime} \mathrm{N}, \\
21^{\circ} 35^{\prime} 22.853^{\prime \prime} \mathrm{E}\end{array}$ & NP & 3 & $\begin{array}{l}\text { Seashore meadow } \\
\text { or dry meadow }\end{array}$ & \\
\hline Aspö & Norpaskäret & $\begin{array}{l}59^{\circ} 53^{\prime} 32.934^{\prime \prime} \mathrm{N}, \\
21^{\circ} 35^{\prime} 10.443^{\prime \prime} \mathrm{E}\end{array}$ & NP & 2 & Stony shore & \\
\hline Knivskär & Knivskär & $\begin{array}{l}60^{\circ} 0^{\prime} 33.786^{\prime \prime} \mathrm{N} \\
21^{\circ} 58^{\prime} 34.264^{\prime \prime} \mathrm{E}\end{array}$ & - & & & Not found 2014 \\
\hline Stenskär & Lilla Tommosskär & $\begin{array}{l}60^{\circ} 3^{\prime} 13.354^{\prime \prime} \mathrm{N} \\
21^{\circ} 58^{\prime} 55.347^{\prime \prime} \mathrm{E}\end{array}$ & - & & & Not found 2014 \\
\hline Vänö & Inre Klovaskär & $\begin{array}{l}59^{\circ} 48^{\prime} 52.548^{\prime \prime} \mathrm{N}, \\
22^{\circ} 9^{\prime} 25.41^{\prime \prime} \mathrm{E}\end{array}$ & - & 7 & $\begin{array}{l}\text { Seashore meadow } \\
\text { or dry meadow }\end{array}$ & Uprooted 2014-2016 \\
\hline Vänö & Söderön, W-skerry & $\begin{array}{l}59^{\circ} 50^{\prime} 33.138^{\prime \prime} \mathrm{N}, \\
22^{\circ} 7^{\prime} 2.437^{\prime \prime} \mathrm{E}\end{array}$ & - & 9 & Stony shore & \\
\hline Vänö & Söderön, W-skerry & $\begin{array}{l}59^{\circ} 50^{\prime} 30.583^{\prime \prime} \mathrm{N}, \\
22^{\circ} 7^{\prime} 4.228^{\prime \prime} \mathrm{E}\end{array}$ & - & 1 & Stony shore & Uprooted 2014-2015 \\
\hline Vänö & Rönnskär & $\begin{array}{l}59^{\circ} 48^{\prime} 18.828^{\prime \prime} \mathrm{N}, \\
22^{\circ} 5^{\prime} 33.857^{\prime \prime} \mathrm{E}\end{array}$ & - & 1 & Stony shore & \\
\hline Vänö & Skeppasskär & $\begin{array}{l}59^{\circ} 48^{\prime} 57.097^{\prime \prime} \mathrm{N}, \\
22^{\circ} 7^{\prime} 46.988^{\prime \prime} \mathrm{E}\end{array}$ & - & 0.5 & Stony shore & Eradicated \\
\hline Vänö & Klobben & $\begin{array}{l}59^{\circ} 50^{\prime} 3.759^{\prime \prime} \mathrm{N} \\
22^{\circ} 13^{\prime} 1.731^{\prime \prime} \mathrm{E}\end{array}$ & NP & 3 & Stony shore & \\
\hline Vänö & Enskär & $\begin{array}{l}59^{\circ} 49^{\prime} 27.334^{\prime \prime} \mathrm{N}, \\
22^{\circ} 7^{\prime} 59.28^{\prime \prime} \mathrm{E}\end{array}$ & NP & 10 & $\begin{array}{l}\text { Seashore meadow } \\
\text { or dry meadow }\end{array}$ & \\
\hline Vänö & $\begin{array}{l}\text { Långmåsgrunden } \\
\text { (eastern) }\end{array}$ & $\begin{array}{l}59^{\circ} 50^{\prime} 33.932^{\prime \prime} \mathrm{N}, \\
22^{\circ} 14^{\prime} 54.265^{\prime \prime} \mathrm{E}\end{array}$ & NP & 49 & $\begin{array}{l}\text { Seashore meadow } \\
\text { or dry meadow }\end{array}$ & Uprooted 2015 \\
\hline Vänö & Stora Buskär & $\begin{array}{l}59^{\circ} 49^{\prime} 28.377^{\prime \prime} \mathrm{N}, \\
22^{\circ} 9^{\prime} 51.635^{\prime \prime} \mathrm{E}\end{array}$ & NP & 15 & $\begin{array}{l}\text { Seashore meadow } \\
\text { or dry meadow }\end{array}$ & $\begin{array}{l}\text { Uprooted 2013-2016, } \\
\text { greatly reduced }\end{array}$ \\
\hline Vänö & Stora Buskär & $\begin{array}{l}59^{\circ} 49^{\prime} 32.099^{\prime \prime} \mathrm{N}, \\
22^{\circ} 10^{\prime} 5.689^{\prime \prime} \mathrm{E}\end{array}$ & NP & 0.5 & Rock outcrop & Uprooted 2013-2016 \\
\hline Vänö & Stora Buskär & $\begin{array}{l}29^{\circ} 10^{\prime} 7.514^{\prime \prime} \mathrm{N} \\
19^{\circ} 41^{\prime} 19.934^{\prime \prime} \mathrm{E}\end{array}$ & NP & 0.5 & $\begin{array}{l}\text { Seashore meadow } \\
\text { or dry meadow }\end{array}$ & Eradicated \\
\hline Vänö & Bredlandet & $\begin{array}{l}59^{\circ} 49^{\prime} 56.253^{\prime \prime} \mathrm{N}, \\
22^{\circ} 10^{\prime} 53.432^{\prime \prime} \mathrm{E}\end{array}$ & - & 18 & Stony shore & \\
\hline Vänö & Ejskär & $\begin{array}{l}59^{\circ} 47^{\prime} 10.82^{\prime \prime} \mathrm{N}, \\
22^{\circ} 12^{\prime} 28.239^{\prime \prime} \mathrm{E}\end{array}$ & NP & 14 & Stony shore & \\
\hline Vänö & Ejskär & $\begin{array}{l}59^{\circ} 47^{\prime} 22.391^{\prime \prime} \mathrm{N}, \\
22^{\circ} 12^{\prime} 30.429^{\prime \prime} \mathrm{E}\end{array}$ & NP & 7 & Stony shore & \\
\hline Vänö & Ejskär & $\begin{array}{l}59^{\circ} 47^{\prime} 22.297^{\prime \prime} \mathrm{N}, \\
22^{\circ} 12^{\prime} 31.406^{\prime \prime} \mathrm{E}\end{array}$ & NP & 2 & Stony shore & \\
\hline Vänö & Krackskär & $\begin{array}{l}59^{\circ} 46^{\prime} 53.438^{\prime \prime} \mathrm{N}, \\
22^{\circ} 12^{\prime} 49.549^{\prime \prime} \mathrm{E}\end{array}$ & - & 15 & Stony shore & \\
\hline Vänö & Örskär (Ejsråsen) & $\begin{array}{l}59^{\circ} 47^{\prime} 16.766^{\prime \prime} \mathrm{N}, \\
22^{\circ} 13^{\prime} 8.804^{\prime \prime} \mathrm{E}\end{array}$ & NP & 1 & Stony shore & Uprooted 2015-2016 \\
\hline Vänö & Rottenskär & $\begin{array}{l}59^{\circ} 47^{\prime} 33.026^{\prime \prime} \mathrm{N}, \\
22^{\circ} 13^{\prime} 2.875^{\prime \prime} \mathrm{E}\end{array}$ & NP & 2 & Stony shore & $\begin{array}{l}\text { Control } 2009 \text { and } \\
\text { uprooted 2015-2016 }\end{array}$ \\
\hline Vänö & Rottenskär & $\begin{array}{l}59^{\circ} 47^{\prime} 32.41^{\prime \prime} \mathrm{N}, \\
22^{\circ} 13^{\prime} 3.734^{\prime \prime} \mathrm{E}\end{array}$ & NP & 0.5 & Dwarf shrub heath & Eradicated \\
\hline Vänö & Örs ören & $\begin{array}{l}59^{\circ} 47^{\prime} 22.975^{\prime \prime} \mathrm{N}, \\
22^{\circ} 13^{\prime} 25.51^{\prime \prime} \mathrm{E}\end{array}$ & NP & 1 & Rock outcrop & Uprooted 2015-2016 \\
\hline
\end{tabular}


ApPENDIX. Continued.

\begin{tabular}{|c|c|c|c|c|c|c|}
\hline Locality & Site & Coordinates & $\begin{array}{c}\text { Conservation } \\
\text { area }\end{array}$ & $\begin{array}{l}\text { Size } \\
{\left[\mathrm{m}^{2}\right]}\end{array}$ & Main habitat type & Note \\
\hline Vänö & Örs ören & $\begin{array}{l}59^{\circ} 47^{\prime} 23.9^{\prime \prime} \mathrm{N}, \\
22^{\circ} 13^{\prime} 26.919^{\prime \prime} \mathrm{E}\end{array}$ & NP & 1 & Stony shore & $\begin{array}{l}\text { Control 2009, } \\
\text { uprooted 2015-2016 }\end{array}$ \\
\hline Vänö & Örs ören & $\begin{array}{l}59^{\circ} 47^{\prime} 24.357^{\prime \prime} \mathrm{N}, \\
22^{\circ} 13^{\prime} 26.147^{\prime \prime} \mathrm{E}\end{array}$ & NP & 1 & Stony shore & $\begin{array}{l}\text { Control 2009, } \\
\text { uprooted 2015-2017 }\end{array}$ \\
\hline Vänö & Örs ören & $\begin{array}{l}59^{\circ} 47^{\prime} 24.298^{\prime \prime} \mathrm{N}, \\
22^{\circ} 13^{\prime} 27.183^{\prime \prime} \mathrm{E}\end{array}$ & NP & 4 & Stony shore & $\begin{array}{l}\text { Control 2009, } \\
\text { uprooted 2015-2018 }\end{array}$ \\
\hline Vänö & Kalkskär & $\begin{array}{l}59^{\circ} 46^{\prime} 35.324^{\prime \prime} \mathrm{N}, \\
22^{\circ} 4^{\prime} 46.044^{\prime \prime} \mathrm{E}\end{array}$ & - & 1 & Rock outcrop & \\
\hline Vänö & Kalkskär & $\begin{array}{l}59^{\circ} 46^{\prime} 35.404^{\prime \prime} \mathrm{N}, \\
22^{\circ} 4^{\prime} 45.583^{\prime \prime} \mathrm{E}\end{array}$ & - & 8 & Dwarf shrub heath & Partly uprooted 2015 \\
\hline Vänö & Kalkskär & $\begin{array}{l}59^{\circ} 46^{\prime} 35.341^{\prime \prime} \mathrm{N}, \\
22^{\circ} 4^{\prime} 46.491^{\prime \prime} \mathrm{E}\end{array}$ & - & 0.5 & Rock outcrop & \\
\hline Vänö & Hemören & $\begin{array}{l}59^{\circ} 52^{\prime} 29.788^{\prime \prime} \mathrm{N}, \\
22^{\circ} 13^{\prime} 10.502^{\prime \prime} \mathrm{E}\end{array}$ & - & 90 & Sandy beach & \\
\hline Vänö & Elvingskär & $\begin{array}{l}59^{\circ} 47^{\prime} 5.97^{\prime \prime} \mathrm{N} \\
22^{\circ} 4^{\prime} 25.916^{\prime \prime} \mathrm{E}\end{array}$ & - & 6 & Rock outcrop & \\
\hline Tunnhamn & $\begin{array}{l}\text { Ängeskärs grunden } \\
\text { S-skerry }\end{array}$ & $\begin{array}{l}59^{\circ} 54^{\prime} 26.973^{\prime \prime} \mathrm{N}, \\
22^{\circ} 8^{\prime} 18.761^{\prime \prime} \mathrm{E}\end{array}$ & - & 13 & Stony shore & \\
\hline Tunnhamn & $\begin{array}{l}\text { Stora Dunskär, E- } \\
\text { skerry }\end{array}$ & $\begin{array}{l}59^{\circ} 53^{\prime} 59.389^{\prime \prime} \mathrm{N}, \\
22^{\circ} 14^{\prime} 36.781^{\prime \prime} \mathrm{E}\end{array}$ & - & 20 & Stony shore & \\
\hline Rosala & Stora Gallbyskäret & $\begin{array}{l}59^{\circ} 47^{\prime} 24.963^{\prime \prime} \mathrm{N}, \\
22^{\circ} 26^{\prime} 39.763^{\prime \prime} \mathrm{E}\end{array}$ & - & 40 & Stony shore & Partly uprooted 2014 \\
\hline Rosala & Lilla Gallbyskäret & $\begin{array}{l}59^{\circ} 47^{\prime} 35.702^{\prime \prime} \mathrm{N}, \\
22^{\circ} 26^{\prime} 35.856^{\prime \prime} \mathrm{E}\end{array}$ & - & 1 & Rock outcrop & Eradicated \\
\hline Rosala & Ljusskär & $\begin{array}{l}59^{\circ} 46^{\prime} 49.116^{\prime \prime} \mathrm{N}, \\
22^{\circ} 26^{\prime} 32.656^{\prime \prime} \mathrm{E}\end{array}$ & - & 6 & Rock outcrop & Eradicated \\
\hline Rosala & Dömmaskär & $\begin{array}{l}59^{\circ} 44^{\prime} 38.523^{\prime \prime} \mathrm{N}, \\
22^{\circ} 30^{\prime} 19.444^{\prime \prime} \mathrm{E}\end{array}$ & - & 4 & Stony shore & Uprooted 2014 \\
\hline Rosala & Dömmaskär & $\begin{array}{l}59^{\circ} 44^{\prime} 41.185^{\prime \prime} \mathrm{N}, \\
22^{\circ} 30^{\prime} 19.663^{\prime \prime} \mathrm{E}\end{array}$ & - & 0.5 & $\begin{array}{l}\text { Seashore meadow } \\
\text { or dry meadow }\end{array}$ & Eradicated \\
\hline Rosala & Måsskäret, S-part & $\begin{array}{l}59^{\circ} 47^{\prime} 48.672^{\prime \prime} \mathrm{N}, \\
22^{\circ} 26^{\prime} 18.862^{\prime \prime} \mathrm{E}\end{array}$ & - & 17 & Stony shore & Uprooted 2015 \\
\hline Rosala & Måsskäret, S-part & $\begin{array}{l}59^{\circ} 47^{\prime} 49.803^{\prime \prime} \mathrm{N}, \\
22^{\circ} 26^{\prime} 12.285^{\prime \prime} \mathrm{E}\end{array}$ & - & 17 & Dwarf shrub heath & Uprooted 2015 \\
\hline Rosala & Måsskäret, N-part & $\begin{array}{l}59^{\circ} 47^{\prime} 52.856^{\prime \prime} \mathrm{N}, \\
22^{\circ} 26^{\prime} 11.674^{\prime \prime} \mathrm{E}\end{array}$ & - & 5 & Rock outcrop & \\
\hline Rosala & Måsskäret, NE-skerry & $\begin{array}{l}59^{\circ} 47^{\prime} 57.241^{\prime \prime} \mathrm{N}, \\
22^{\circ} 26^{\prime} 13.065^{\prime \prime} \mathrm{E}\end{array}$ & - & 2 & Rock outcrop & \\
\hline Rosala & $\begin{array}{l}\text { Nordanvädergrundet, } \\
\text { N-skerry }\end{array}$ & $\begin{array}{l}59^{\circ} 49^{\prime} 41.752^{\prime \prime} \mathrm{N}, \\
22^{\circ} 31^{\prime} 13.214^{\prime \prime} \mathrm{E}\end{array}$ & - & 5 & Stony shore & \\
\hline Rosala & Örö, west shore & $\begin{array}{l}59^{\circ} 48^{\prime} 12.131^{\prime \prime} \mathrm{N}, \\
22^{\circ} 18^{\prime} 35.666^{\prime \prime} \mathrm{E}\end{array}$ & NP & 2000 & Sandy beach & $\begin{array}{l}\text { Control since } 2008, \\
\text { greatly reduced }\end{array}$ \\
\hline Rosala & Örö, west shore & $\begin{array}{l}59^{\circ} 48^{\prime} 21.638^{\prime \prime} \mathrm{N}, \\
22^{\circ} 18^{\prime} 42.555^{\prime \prime} \mathrm{E}\end{array}$ & NP & 2500 & Sandy beach & $\begin{array}{l}\text { Documented } 1963, \\
\text { control since } 2008, \\
\text { almost eradicated }\end{array}$ \\
\hline Rosala & Örö, Balget & $\begin{array}{l}59^{\circ} 48^{\prime} 26.197^{\prime \prime} \mathrm{N}, \\
22^{\circ} 19^{\prime} 32.572^{\prime \prime} \mathrm{E}\end{array}$ & NP & 16 & Sandy beach & Control 2015 \\
\hline Rosala & Örö, Solkuro & $\begin{array}{l}59^{\circ} 48^{\prime} 9.88^{\prime \prime} \mathrm{N} \\
22^{\circ} 19^{\prime} 1.497^{\prime \prime} \mathrm{E}\end{array}$ & NP & 7 & Stony shore & $\begin{array}{l}\text { Grew partly under } \\
\text { black alders, control } \\
2015\end{array}$ \\
\hline Rosala & Syllskären & $\begin{array}{l}59^{\circ} 47^{\prime} 47.236^{\prime \prime} \mathrm{N}, \\
22^{\circ} 18^{\prime} 41.066^{\prime \prime} \mathrm{E}\end{array}$ & NP & 0.5 & Dwarf shrub heath & \\
\hline Rosala & Arlanden & $\begin{array}{l}59^{\circ} 48^{\prime} 8.608^{\prime \prime} \mathrm{N}, \\
22^{\circ} 20^{\prime} 37.737^{\prime \prime} \mathrm{E}\end{array}$ & NP & 25 & $\begin{array}{l}\text { Seashore meadow } \\
\text { or dry meadow }\end{array}$ & \\
\hline
\end{tabular}


ApPENDIX. Continued.

\begin{tabular}{|c|c|c|c|c|c|c|}
\hline Locality & Site & Coordinates & \begin{tabular}{|c|}
$\begin{array}{c}\text { Conservation } \\
\text { area }\end{array}$ \\
\end{tabular} & $\begin{array}{l}\text { Size } \\
{\left[\mathrm{m}^{2}\right]}\end{array}$ & Main habitat type & Note \\
\hline Rosala & Stengrundet & $\begin{array}{l}59^{\circ} 52^{\prime} 20.142^{\prime \prime} \mathrm{N} \\
22^{\circ} 20^{\prime} 28.026^{\prime \prime} \mathrm{E}\end{array}$ & - & 126 & Sandy beach & \\
\hline Rosala & Måskobben & $\begin{array}{l}59^{\circ} 50^{\prime} 23.308^{\prime \prime} \mathrm{N} \\
22^{\circ} 31^{\prime} 39.9^{\prime \prime} \mathrm{E}\end{array}$ & - & 0.5 & Rock outcrop & Uprooted 2016 \\
\hline Rosala & $\begin{array}{l}\begin{array}{l}\text { Lammgrunden } \\
\text { (northern) }\end{array} \\
\end{array}$ & $\begin{array}{l}59^{\circ} 48^{\prime} 25.609^{\prime \prime} \mathrm{N} \\
22^{\circ} 27^{\prime} 50.684^{\prime \prime} \mathrm{E}\end{array}$ & - & 1 & Rock outcrop & \\
\hline Rosala & $\begin{array}{l}\text { Lammgrunden } \\
\text { (northern) }\end{array}$ & $\begin{array}{l}59^{\circ} 48^{\prime} 27.058^{\prime \prime} \mathrm{N} \\
22^{\circ} 27^{\prime} 39.244^{\prime \prime} \mathrm{E}\end{array}$ & - & 6 & Dwarf shrub heath & \\
\hline Rosala & $\begin{array}{l}\text { Lammgrunden } \\
\text { (southern) }\end{array}$ & $\begin{array}{l}59^{\circ} 48^{\prime} 18.424^{\prime \prime} \mathrm{N} \\
22^{\circ} 27^{\prime} 34.767^{\prime \prime} \mathrm{E}\end{array}$ & - & 13 & $\begin{array}{l}\text { Seashore meadow } \\
\text { or dry meadow }\end{array}$ & \\
\hline Rosala & $\begin{array}{l}\text { Lammgrunden } \\
\text { (southern) }\end{array}$ & $\begin{array}{l}59^{\circ} 48^{\prime} 18.138^{\prime \prime} \mathrm{N} \\
22^{\circ} 27^{\prime} 33.971^{\prime \prime} \mathrm{E}\end{array}$ & - & 2 & Rock outcrop & \\
\hline Rosala & Äggeskäret & $\begin{array}{l}59^{\circ} 48^{\prime} 11.746^{\prime \prime} \mathrm{N} \\
22^{\circ} 27^{\prime} 25.078^{\prime \prime} \mathrm{E}\end{array}$ & - & 3 & Rock outcrop & \\
\hline Rosala & Äggeskäret & $\begin{array}{l}59^{\circ} 48^{\prime} 14.392^{\prime \prime} \mathrm{N} \\
22^{\circ} 27^{\prime} 16.431^{\prime \prime} \mathrm{E}\end{array}$ & - & 7 & Stony shore & \\
\hline Rosala & Äggeskäret & $\begin{array}{l}59^{\circ} 48^{\prime} 11.578^{\prime \prime} \mathrm{N} \\
22^{\circ} 27^{\prime} 24.908^{\prime \prime} \mathrm{E}\end{array}$ & - & 15 & Rock outcrop & \\
\hline Rosala & Gråskäret & $\begin{array}{l}59^{\circ} 47^{\prime} 56.494^{\prime \prime} \mathrm{N} \\
22^{\circ} 19^{\prime} 37.381^{\prime \prime} \mathrm{E}\end{array}$ & NP & 20 & $\begin{array}{l}\text { Seashore meadow } \\
\text { or dry meadow }\end{array}$ & \\
\hline Rosala & Slamrorna, W-skerry & $\begin{array}{l}59^{\circ} 48^{\prime} 51.219^{\prime \prime} \mathrm{N} \\
22^{\circ} 28^{\prime} 5.06^{\prime \prime} \mathrm{E}\end{array}$ & - & 6 & Dwarf shrub heath & \\
\hline Rosala & Kuggskär & $\begin{array}{l}59^{\circ} 46^{\prime} 38.152^{\prime \prime} \mathrm{N} \\
22^{\circ} 22^{\prime} 26.841^{\prime \prime} \mathrm{E}\end{array}$ & PNR & 17 & Dwarf shrub heath & \\
\hline Rosala & Kuggskär, S-shore & $\begin{array}{l}59^{\circ} 46^{\prime} 35.857^{\prime \prime} \mathrm{N} \\
22^{\circ} 22^{\prime} 23.243^{\prime \prime} \mathrm{E}\end{array}$ & PNR & 7 & Stony shore & \\
\hline Rosala & Kuggskär & $\begin{array}{l}59^{\circ} 46^{\prime} 42.384^{\prime \prime} \mathrm{N} \\
22^{\circ} 22^{\prime} 36.717^{\prime \prime} \mathrm{E}\end{array}$ & PNR & 31 & $\begin{array}{l}\text { Seashore meadow } \\
\text { or dry meadow }\end{array}$ & \\
\hline Rosala & Kuggskär & $\begin{array}{l}59^{\circ} 46^{\prime} 42.201^{\prime \prime} \mathrm{N} \\
22^{\circ} 22^{\prime} 37.962^{\prime \prime} \mathrm{E}\end{array}$ & PNR & 11 & $\begin{array}{l}\text { Seashore meadow } \\
\text { or dry meadow }\end{array}$ & \\
\hline Rosala & Kuggskär, W-shore & $\begin{array}{l}59^{\circ} 46^{\prime} 57.604^{\prime \prime} \mathrm{N} \\
22^{\circ} 22^{\prime} 17.659^{\prime \prime} \mathrm{E}\end{array}$ & PNR & 24 & $\begin{array}{l}\text { Seashore meadow } \\
\text { or dry meadow }\end{array}$ & $\begin{array}{l}\text { Found } 1967 \text {, partly } \\
\text { uprooted } 2012\end{array}$ \\
\hline Rosala & Kuggskär, E-shore & $\begin{array}{l}59^{\circ} 46^{\prime} 49.864^{\prime \prime} \mathrm{N} \\
22^{\circ} 22^{\prime} 46.914^{\prime \prime} \mathrm{E}\end{array}$ & - & 40 & $\begin{array}{l}\text { Seashore meadow } \\
\text { or dry meadow }\end{array}$ & \\
\hline Rosala & Kuggskär, E-shore & $\begin{array}{l}59^{\circ} 46^{\prime} 48.993^{\prime \prime} \mathrm{N} \\
22^{\circ} 22^{\prime} 45.173^{\prime \prime} \mathrm{E}\end{array}$ & - & 54 & $\begin{array}{l}\text { Seashore meadow } \\
\text { or dry meadow }\end{array}$ & \\
\hline Rosala & Kuggskär, W-shore & $\begin{array}{l}59^{\circ} 46^{\prime} 56.884^{\prime \prime} \mathrm{N} \\
22^{\circ} 22^{\prime} 16.539^{\prime \prime} \mathrm{E}\end{array}$ & PNR & 7 & Stony shore & \\
\hline Rosala & Kuggskär, N-shore & $\begin{array}{l}59^{\circ} 47^{\prime} 0.442^{\prime \prime} \mathrm{N}, \\
22^{\circ} 22^{\prime} 32.994^{\prime \prime} \mathrm{E}\end{array}$ & PNR & 0.5 & Stony shore & \\
\hline Hitis & Yttersta Hamnskär & $\begin{array}{l}59^{\circ} 50^{\prime} 32.334^{\prime \prime} \mathrm{N} \\
22^{\circ} 37^{\prime} 35.251^{\prime \prime} \mathrm{E}\end{array}$ & - & 0.5 & Rock outcrop & Uprooted 2015 \\
\hline Hitis & Flintskär & $\begin{array}{l}59^{\circ} 50^{\prime} 4.039^{\prime \prime} \mathrm{N}, \\
22^{\circ} 47^{\prime} 18.634^{\prime \prime} \mathrm{E}\end{array}$ & - & 3 & Stony shore & \\
\hline Hitis & Salskären & $\begin{array}{l}59^{\circ} 50^{\prime} 34.879^{\prime \prime} \mathrm{N} \\
22^{\circ} 38^{\prime} 45.404^{\prime \prime} \mathrm{E}\end{array}$ & - & 9 & Stony shore & \\
\hline Hitis & Fågelskäret SE-skerry & $\begin{array}{l}59^{\circ} 53^{\prime} 33.634^{\prime \prime} \mathrm{N} \\
22^{\circ} 37^{\prime} 13.84^{\prime \prime} \mathrm{E}\end{array}$ & - & 3 & Stony shore & \\
\hline Hitis & Morgonlandet & $\begin{array}{l}59^{\circ} 45^{\prime} 33.976^{\prime \prime} \mathrm{N} \\
22^{\circ} 42^{\prime} 16.684^{\prime \prime} \mathrm{E}\end{array}$ & NP & 0.5 & Rock outcrop & \\
\hline Hitis & Morgonlandet & $\begin{array}{l}59^{\circ} 45^{\prime} 34.37^{\prime \prime} \mathrm{N}, \\
22^{\circ} 42^{\prime} 15.864^{\prime \prime} \mathrm{E}\end{array}$ & NP & 6 & Rock outcrop & $\begin{array}{l}\text { Grew partly under } \\
\text { black alders }\end{array}$ \\
\hline Hitis & $\begin{array}{l}\text { Lukargrunden (sout- } \\
\text { hern) }\end{array}$ & $\begin{array}{l}59^{\circ} 48^{\prime} 12.621^{\prime \prime} \mathrm{N}, \\
22^{\circ} 26^{\prime} 51.492^{\prime \prime} \mathrm{E}\end{array}$ & - & 10 & Rock outcrop & \\
\hline
\end{tabular}


ApPENDIX. Continued.

\begin{tabular}{|c|c|c|c|c|c|c|}
\hline Locality & Site & Coordinates & $\begin{array}{c}\text { Conservation } \\
\text { area }\end{array}$ & $\begin{array}{l}\text { Size } \\
{\left[\mathrm{m}^{2}\right]}\end{array}$ & Main habitat type & Note \\
\hline Hitis & $\begin{array}{l}\text { Lukargrunden } \\
\text { (southern) }\end{array}$ & $\begin{array}{l}59^{\circ} 48^{\prime} 12.243^{\prime \prime} \mathrm{N} \\
22^{\circ} 26^{\prime} 50.837^{\prime \prime} \mathrm{E}\end{array}$ & - & 0.5 & Rock outcrop & \\
\hline Hitis & Mellangrundet & $\begin{array}{l}59^{\circ} 53^{\prime} 11.07^{\prime \prime} \mathrm{N}, \\
22^{\circ} 36^{\prime} 42.495^{\prime \prime} \mathrm{E}\end{array}$ & - & 4 & $\begin{array}{l}\text { Seashore meadow } \\
\text { or dry meadow }\end{array}$ & \\
\hline Hitis & Rysskärs ören & $\begin{array}{l}59^{\circ} 53^{\prime} 33.377^{\prime \prime} \mathrm{N} \\
22^{\circ} 38^{\prime} 33.795^{\prime \prime} \mathrm{E}\end{array}$ & - & 1 & Stony shore & \\
\hline Hitis & Rysskärs ören & $\begin{array}{l}59^{\circ} 53^{\prime} 33.813^{\prime \prime} \mathrm{N} \\
22^{\circ} 38^{\prime} 33.287^{\prime \prime} \mathrm{E}\end{array}$ & - & 4 & Dwarf shrub heath & \\
\hline Hitis & Rysskärs ören & $\begin{array}{l}59^{\circ} 53^{\prime} 33.536^{\prime \prime} \mathrm{N} \\
22^{\circ} 38^{\prime} 33.71^{\prime \prime} \mathrm{E}\end{array}$ & - & 6 & Stony shore & \\
\hline Hitis & Rysskobben & $\begin{array}{l}59^{\circ} 53^{\prime} 50.609^{\prime \prime} \mathrm{N} \\
22^{\circ} 40^{\prime} 57.612^{\prime \prime} \mathrm{E}\end{array}$ & - & 3 & $\begin{array}{l}\text { Seashore meadow } \\
\text { or dry meadow }\end{array}$ & \\
\hline Hitis & Rysskobben, W-skerry & $\begin{array}{l}59^{\circ} 53^{\prime} 53.248^{\prime \prime} \mathrm{N} \\
22^{\circ} 40^{\prime} 52.245^{\prime \prime} \mathrm{E}\end{array}$ & - & 17 & $\begin{array}{l}\text { Seashore meadow } \\
\text { or dry meadow }\end{array}$ & \\
\hline Hitis & Bengtskär & $\begin{array}{l}59^{\circ} 43^{\prime} 25.095^{\prime \prime} \mathrm{N} \\
22^{\circ} 29^{\prime} 55.93^{\prime \prime} \mathrm{E}\end{array}$ & - & 1 & Rock outcrop & \\
\hline Hitis & Halsskäret & $\begin{array}{l}59^{\circ} 53^{\prime} 3.699^{\prime \prime} \mathrm{N}, \\
22^{\circ} 45^{\prime} 39.451^{\prime \prime} \mathrm{E}\end{array}$ & - & 6 & $\begin{array}{l}\text { Seashore meadow } \\
\text { or dry meadow }\end{array}$ & \\
\hline Hitis & Mankomskäret & $\begin{array}{l}59^{\circ} 49^{\prime} 17.953^{\prime \prime} \mathrm{N}, \\
22^{\circ} 27^{\prime} 37.0^{\prime \prime} \mathrm{E}\end{array}$ & - & 1 & Rock outcrop & \\
\hline Hitis & Kråklandet, S-skerry & $\begin{array}{l}59^{\circ} 53^{\prime} 37.779^{\prime \prime} \mathrm{N}, \\
22^{\circ} 37^{\prime} 33.387^{\prime \prime} \mathrm{E}\end{array}$ & - & 5 & $\begin{array}{l}\text { Seashore meadow } \\
\text { or dry meadow }\end{array}$ & \\
\hline Hitis & Norra Stenharun & $\begin{array}{l}59^{\circ} 53^{\prime} 28.983^{\prime \prime} \mathrm{N}, \\
22^{\circ} 39^{\prime} 26.855^{\prime \prime} \mathrm{E}\end{array}$ & - & 7 & Stony shore & \\
\hline Hitis & Prackgrundet & $\begin{array}{l}59^{\circ} 53^{\prime} 15.233^{\prime \prime} \mathrm{N}, \\
22^{\circ} 36^{\prime} 17.927^{\prime \prime} \mathrm{E}\end{array}$ & - & 24 & $\begin{array}{l}\text { Seashore meadow } \\
\text { or dry meadow }\end{array}$ & \\
\hline Hitis & Tjukan & $\begin{array}{l}59^{\circ} 52^{\prime} 56.712^{\prime \prime} \mathrm{N}, \\
22^{\circ} 44^{\prime} 4.422^{\prime \prime} \mathrm{E}\end{array}$ & - & 1 & Stony shore & \\
\hline Hitis & Tjukan & $\begin{array}{l}59^{\circ} 52^{\prime} 59.372^{\prime \prime} \mathrm{N} \\
22^{\circ} 43^{\prime} 54.682^{\prime \prime} \mathrm{E}\end{array}$ & - & 2 & $\begin{array}{l}\text { Seashore meadow } \\
\text { or dry meadow }\end{array}$ & \\
\hline Hitis & Nickelskäret & $\begin{array}{l}59^{\circ} 52^{\prime} 1.655^{\prime \prime} \mathrm{N}, \\
22^{\circ} 36^{\prime} 43.357^{\prime \prime} \mathrm{E}\end{array}$ & - & 0.5 & Stony shore & Uprooted 2015 \\
\hline Hitis & Hamnskäret & $\begin{array}{l}59^{\circ} 51^{\prime} 41.106^{\prime \prime} \mathrm{N}, \\
22^{\circ} 36^{\prime} 28.825^{\prime \prime} \mathrm{E}\end{array}$ & - & 1 & Stony shore & Partly uprooted 2015 \\
\hline
\end{tabular}

